TRANSACTIONS OF THE

AMERICAN MATHEMATICAL SOCIETY

Volume 350, Number 3, March 1998, Pages 857-887

S 0002-9947(98)01659-6

\title{
POISSON TRANSFORMS ON VECTOR BUNDLES
}

\author{
AN YANG
}

\begin{abstract}
Let $G$ be a connected real semisimple Lie group with finite center, and $K$ a maximal compact subgroup of $G$. Let $(\tau, V)$ be an irreducible unitary representation of $K$, and $G \times_{K} V$ the associated vector bundle. In the algebra of invariant differential operators on $G \times_{K} V$ the center of the universal enveloping algebra of $\operatorname{Lie}(G)$ induces a certain commutative subalgebra $Z_{\tau}$. We are able to determine the characters of $Z_{\tau}$. Given such a character we define a Poisson transform from certain principal series representations to the corresponding space of joint eigensections. We prove that for most of the characters this map is a bijection, generalizing a famous conjecture by Helgason which corresponds to $\tau$ the trivial representation.
\end{abstract}

\section{INTRODUCTION}

Let $G$ be a connected real semisimple Lie group with finite center, and $K$ a maximal compact subgroup of $G$. Then $G / K$ is a Riemannian symmetric space of noncompact type. We fix an Iwasawa decomposition $G=K A N$. Let $M$ be the centralizer of $A$ in $K$. Let $\mathfrak{g}$ and $\mathfrak{a}$ be the Lie algebras of $G$ and $A$, respectively, and $\Sigma(\mathfrak{g}, \mathfrak{a})$ the root system for $\mathfrak{g}, \mathfrak{a}$. Let $\Sigma^{+}(\mathfrak{g}, \mathfrak{a})$ be the positive roots in $\Sigma(\mathfrak{g}, \mathfrak{a})$ for the ordering given by $N$. Let $D(G / K)$ be the algebra of invariant differential operators on $G / K$. It is well-known that the characters of $D(G / K)$ are parametrized by $\lambda \in \mathfrak{a}_{\mathbb{C}}^{*}$, the complex dual space of $\mathfrak{a}$. Let $\mathcal{E}_{\lambda}(G / K)$ denote the space of joint eigenfunctions corresponding to $\lambda$. We write $g=\mathrm{k}(g) \operatorname{expH}(g) \mathrm{n}(g)$ for each $g \in G$ according to $G=K A N$. For each $\phi \in C^{\infty}(K / M)$ we define $P_{\lambda} \phi \in C^{\infty}(G / K)$ by

$$
P_{\lambda} \phi(g)=\int_{K} \phi(k) e^{-(\lambda+\rho) \mathrm{H}\left(g^{-1} k\right)} d k .
$$

Here $\rho$ is the half sum of $\Sigma^{+}(\mathfrak{g}, \mathfrak{a})$ (including multiplicities). It turns out that $P_{\lambda} \phi \in$ $\mathcal{E}_{\lambda}(G / K)$. One can easily extend the definition of $P_{\lambda}$ to the space $D^{\prime}(K / M)$ (resp. $\left.A^{\prime}(K / M)\right)$ of distributions (resp. analytic functionals) on $K / M$. In this paragraph we fix $\lambda \in \mathfrak{a}_{\mathbb{C}}^{*}$ such that $2\langle\lambda, \alpha\rangle /\langle\alpha, \alpha\rangle$ is not in $-\mathbb{N}-\{0\}$, for each $\alpha \in \Sigma^{+}(\mathfrak{g}, \mathfrak{a})$. It is proved by Helgason [Helg2] that $P_{\lambda}$ defines a bijection from $C^{\infty}(K / M)_{K \text {-finite }}$ onto $\mathcal{E}_{\lambda}(G / K)_{K \text {-finite. }}$ He also proves in the rank one case $P_{\lambda}$ is a bijection from $A^{\prime}(K / M)$ onto $\mathcal{E}_{\lambda}(G / K)$. He then conjectured this should be true for the higher rank case. The conjecture was eventually proved by six Japanese mathematicians in [KKMOOT]. It should be mentioned that a representation theoretic proof by Schmid, starting from the $K$-finite result, is indicated in [Sch]. Lewis, then a student of Helgason, made the following observation: Let $\mathcal{E}_{\lambda}^{*}(G / K)$ be the subspace of $\mathcal{E}_{\lambda}(G / K)$ where each element increases at most exponentially (see $\S 2$ for definition);

Received by the editors September 28, 1994 and, in revised form, January 30, 1995.

1991 Mathematics Subject Classification. Primary 22E46; Secondary 43A85.

(C)1998 American Mathematical Society 
then $P_{\lambda}$ maps $D^{\prime}(K / M)$ into $\mathcal{E}_{\lambda}^{*}(G / K)$. He was able to prove in the rank one case $P_{\lambda}$ is a bijection from $D^{\prime}(K / M)$ onto $\mathcal{E}_{\lambda}^{*}(G / K)$. See [Lew]. This result has been generalized to the higher rank case by Oshima and Sekiguchi [OS]. There is an alternative and independent proof by Wallach [Wall1]. By refining Wallach's idea van den Ban and Schlichtkrull have a third proof in [BS]. They define $\mathcal{E}_{\lambda}^{\infty}(G / K)$ as the subspace of $\mathcal{E}_{\lambda}(G / K)$ where each element and its derivatives increase at most exponentially (uniformly). Then they prove $P_{\lambda}$ is a bijection from $C^{\infty}(K / M)$ onto $\mathcal{E}_{\lambda}^{\infty}(G / K)$. The bijectivity of $P_{\lambda}$ from $D^{\prime}(K / M)$ to $\mathcal{E}_{\lambda}^{*}(G / K)$ follows easily.

Let $(\tau, V)$ be an irreducible unitary representation of $K$. Let $G \times_{K} V$ be the associated vector bundle over $G / K$. The space of smooth sections of this vector bundle can be identified with

$$
C^{\infty} \operatorname{Ind}_{K}^{G}(\tau)=\left\{f \in C^{\infty}(G, V) \mid f(g k)=\tau\left(k^{-1}\right) f(g), \forall g \in G, \forall k \in K\right\} .
$$

Let $D_{\tau}$ denote the algebra of invariant differential operators on $C^{\infty} \operatorname{Ind}_{K}^{G}(\tau)$. Notice when $(\tau, V)$ is the trivial representation we go back to the previous case. In the case where $\operatorname{dim} V=1, D_{\tau}$ is commutative and its characters can be parameterized by $\lambda \in \mathfrak{a}_{\mathbb{C}}^{*}$. In [Shim] Shimeno is able to characterize the joint eigenspace of $D_{\tau}$ in terms of a Poisson transform for most of $\lambda$. Gaillard's results about the eigenforms of the Laplacian on hyperbolic spaces are illuminating. They show considerable variety even for a simple space. See [Ga] for details. van der Ven [Ven] considers vector-valued Poisson transforms in the rank one case, extending Gaillard's results. His emphasis, however, is on the singular eigenvalues. Minemura [Min] studies the properties of $D_{\tau}$ and obtains a result on the dimension of the spherical eigensections.

One of the difficulties people run into when trying to generalize the classical results is the complexity of $D_{\tau}$, in particular its noncommutativity. The remedy used was either a condition on $\tau$ or a condition on $(G / K)$. We put a mild condition on $\mathfrak{g}$ (see beginning of $\S 4$ ) but no restriction on $\tau$. We replace $D_{\tau}$ with a subalgebra $Z_{\tau}$ coming from $\mathcal{Z}(\mathfrak{g})$, the center of the universal enveloping algebra of $\mathfrak{g}_{\mathbb{C}}$. Then we are able to determine the characters of $Z_{\tau}$. It turns out they are given by $\lambda-\Lambda$, where $\lambda \in \mathfrak{a}_{\mathbb{C}}^{*}$, and $\Lambda$ is given by the infinitesimal character of an irreducible representation of $M$ contained in $\tau$ (see Proposition 1.11).

Let $V$ be the representation space of $\tau$, and

$$
V=\bigoplus_{\sigma \in \widehat{M}} V(\sigma)
$$

the isotypic decomposition of $V$ into $M$-isotypic parts. We say $\sigma \in \tau$ if $V(\sigma) \neq 0$. Write

$$
V(\Lambda)=\bigoplus_{\sigma \in \tau, \Lambda_{\sigma}=\Lambda} V(\sigma) .
$$

Here $\Lambda_{\sigma}$ is given by the infinitesimal character of $\sigma$. Let $\tau(\Lambda)$ be the restriction of $\tau$ to $M$ with representation space $V(\Lambda)$. We define a Poisson transform (see $\S 1$ for definition)

$$
P_{\lambda}: C^{\infty} \operatorname{Ind}_{M A N}^{G}(\tau(\Lambda) \otimes(-\lambda) \otimes 1) \rightarrow \mathcal{E}_{\lambda-\Lambda}^{\infty} \operatorname{Ind}_{K}^{G}(\tau)
$$

by

$$
P_{\lambda} \phi(g)=\int_{K} \tau(k) \phi(g k) d k .
$$


Here $C^{\infty} \operatorname{Ind}_{M A N}^{G}(\tau(\Lambda) \otimes(-\lambda) \otimes 1)$ is the space defined by

$$
\left\{\phi \in C^{\infty}(G, V(\Lambda)) \mid \phi(\text { gman })=a^{\lambda-\rho} \tau\left(m^{-1}\right) \phi(g)\right\},
$$

and $\mathcal{E}_{\lambda-\Lambda}^{\infty} \operatorname{Ind}_{K}^{G}(\tau)$ the subspace of the total eigenspace where each element and its derivatives increase at most exponentially (uniformly). Let $C(\lambda)$ be the generalized Harish-Chandra $C$-function corresponding to $\tau$ (Proposition 2.3), $C_{0}(\lambda)$ the restriction of $C(\lambda)$ to $V(\Lambda)$, and $\Sigma\left(\mathfrak{g}_{\mathbb{C}}, \mathfrak{h}_{\mathbb{C}}\right)$ as defined after Remark 1.5.

Theorem. Let $\lambda-\Lambda \in \mathfrak{h}_{\mathbb{C}}^{*}$, satisfying the following conditions:

(i) $2\langle\lambda-\Lambda, \alpha\rangle /\langle\alpha, \alpha\rangle \notin \mathbb{Z}, \forall \alpha \in \Sigma\left(\mathfrak{g}_{\mathbb{C}}, \mathfrak{h}_{\mathbb{C}}\right)$, with $\alpha \mid \mathfrak{a} \neq 0$;

(ii) $2\langle\lambda, \beta\rangle /\langle\beta, \beta\rangle \notin-\mathbb{N}, \forall \beta \in \Sigma^{+}(\mathfrak{g}, \mathfrak{a})$;

(iii) $\operatorname{det} C_{0}(\lambda) \neq 0$.

Then $P_{\lambda}$ is a bijection.

This generalizes the result of van den and Ban and Schlichtkrull mentioned above which corresponds to $\tau$ the trivial representation.

We have similar results about distributions and $K$-finite sections, generalizing the above-mentioned results for $\tau$ trivial.

The main idea in the proof is to generalize the theory of asymptotic expansions developed in [Ban] and [BS]. By invoking Casselman's deep result [Ca] on globalization of Harish-Chandra modules, one might simplify our argument somehow. But we prefer a self-contained account. Besides, we think the theory of asymptotic expansions developed here is of interest on its own.

The paper is organized as follows: in Section 1 we study the invariant differential operators on $G \times_{K} V$. In Section 2 we introduce some function spaces on $G$. In Section 3 we state some results on the asymptotic expansion of an eigensection. In Section 4 we study the algebraic structure of a $(\mathfrak{g}, K)$-module. In Sections 5 and 6 we prove the results stated in Section 3. In Section 7 we study the leading terms of the asymptotic expansion. In Section 8 we give an inversion formula to the Poisson transform. In Sections 9 and 10 we extend the Poisson transform to vector-valued distributions.

\section{NOtATIONS AND PRELIMINARIES}

Let $G$ be a connected real semisimple Lie group with finite center and $K$ a maximal compact subgroup of $G$. Then $G / K$ is a Riemannian symmetric space. We fix an Iwasawa decomposition $G=K A N$, and let $M$ be the centralizer of $A$ in $K, M^{\prime}$ the normalizer of $A$ in $K, W=M^{\prime} / M$ the Weyl group. Let $\mathfrak{g}, \mathfrak{k}, \mathfrak{a}, \mathfrak{n}$, and $\mathfrak{m}$ be the corresponding Lie algebras of $G, K, A, N$, and $M$, respectively, and $U(\mathfrak{g}), U(\mathfrak{k}), U(\mathfrak{a}), U(\mathfrak{n})$, and $U(\mathfrak{m})$ the corresponding universal enveloping algebras of the complexified Lie algebras. Let $\Sigma(\mathfrak{g}, \mathfrak{a})$ be the restricted root system for $(\mathfrak{g}, \mathfrak{a})$, and $\Delta=\left\{\alpha_{1}, \ldots, \alpha_{r}\right\}$ the set of simple roots for the ordering of $\Sigma(\mathfrak{g}, \mathfrak{a})$ given by $N$. Let $\mathcal{Z}(\mathfrak{g})$ be the center of $U(\mathfrak{g})$. If $g \in G$ we write $g=\mathrm{k}(g) \exp \mathrm{H}(g) \mathrm{n}(g)$ according to $G=K A N$.

Fix once and for all an irreducible unitary representation $(\tau, V)$ of $K$. Denote by $G \times_{K} V$ the associated vector bundle. Then the space of its smooth sections may be identified with the following space:

$$
C^{\infty} \operatorname{Ind}_{K}^{G}(\tau)=\left\{f \in C^{\infty}(G, V) \mid f(g k)=\tau(k)^{-1} f(g), \forall g \in G, \forall k \in K\right\} .
$$

Let $D_{\tau}$ denote the algebra of differential operators on $C^{\infty} \operatorname{Ind}_{K}^{G}(\tau)$ that commute with the left translations by elements of $G$. The remainder of this section will be 
devoted to the study of this algebra. First for each $f \in C^{\infty}(G, V)$ and $X \in \mathfrak{g}$ we define $L_{X} f$ and $R_{X} f$ as follows:

$$
\begin{aligned}
L_{X} f(g) & =\left.\left(\frac{d}{d t} f(\exp (-t X) g)\right)\right|_{t=0}, \\
R_{X} f(g) & =\left.\left(\frac{d}{d t} f(g \exp t X)\right)\right|_{t=0}, \quad \forall g \in G .
\end{aligned}
$$

Then $L$ and $R$ define two representations of $\mathfrak{g}$ which we extend to representations of $U(\mathfrak{g})$. Let $\operatorname{End}(V)$ denote the space of linear maps from $V$ to itself. Then $U(\mathfrak{g}) \otimes \operatorname{End}(V)$ is an associative algebra with the natural multiplication. Let $I(\tau)$ be the left ideal of $U(\mathfrak{g}) \otimes \operatorname{End}(V)$ generated by $\{X \otimes 1+1 \otimes \tau(X) \mid X \in \mathfrak{k}$. $\}$.

Proposition 1.1. With the above notations, we have

$$
U(\mathfrak{g}) \otimes \operatorname{End}(V)=(U(\mathfrak{a}) \otimes \operatorname{End}(V)) \oplus(\mathfrak{n} U(\mathfrak{g}) \otimes \operatorname{End}(V)+I(\tau)) .
$$

Proof. It suffices to show the left-hand side is contained in the right-hand side. Suppose $u \otimes T \in U(\mathfrak{g}) \otimes \operatorname{End}(V)$. By Poincaré-Birkhoff-Witt we can assume $u=$ $u_{1} u_{2} u_{3}$, where $u_{1} \in U(\mathfrak{n}), u_{2} \in U(\mathfrak{a})$, and $u_{3} \in U(\mathfrak{k})$. If $u_{1} \in \mathfrak{n} U(\mathfrak{n})$ then $u \otimes$ $T \in \mathfrak{n} U(\mathfrak{g}) \otimes \operatorname{End}(V)$. So we can assume $u=u_{2} u_{3}$, where $u_{2} \in U(\mathfrak{a})$, and $u_{3} \in$ $U(\mathfrak{k})$. Let $u_{3}=X_{1} \cdots X_{j}$, for $X_{1}, \ldots, X_{j} \in \mathfrak{k}$. It is easy to show $u_{2} u_{3} \otimes T \in$ $(U(\mathfrak{a}) \otimes \operatorname{End}(V))+I(\tau)$ by induction on $j$.

Define a $K$-action on $U(\mathfrak{g}) \otimes \operatorname{End}(V)$ by

$$
k(X \otimes T)=\operatorname{Ad}(k) X \otimes \tau(k) T \tau(k)^{-1},
$$

for each $k \in K$. Let $(U(\mathfrak{g}) \otimes \operatorname{End}(V))^{K}$ be the fixed elements.

Proposition 1.2. Let $\Gamma_{1}: U(\mathfrak{g}) \otimes \operatorname{End}(V) \rightarrow U(\mathfrak{a}) \otimes \operatorname{End}(V)$ be the projection map according to the decomposition in Proposition 1.1. Then $\Gamma_{1}$ is a homomorphism from $(U(\mathfrak{g}) \otimes \operatorname{End}(V))^{K}$ into $U(\mathfrak{a}) \otimes \operatorname{End}_{M}(V)$, where

$$
\operatorname{End}_{M}(V)=\{T \in \operatorname{End}(V) \mid \tau(m) T=T \tau(m), \forall m \in M\} .
$$

Proof. Since $M$ preserves $\mathfrak{n}$, it is easy to see $\Gamma_{1}$ maps $(U(\mathfrak{g}) \otimes \operatorname{End}(V))^{K}$ into $U(\mathfrak{a}) \otimes \operatorname{End}_{M}(V)$. We now check $\Gamma_{1}$ is a homomorphism.

Suppose $D_{1}, D_{2} \in(U(\mathfrak{g}) \otimes \operatorname{End}(V))^{K}$. Then

$$
D_{1}-\Gamma_{1}\left(D_{1}\right) \in \mathfrak{n} U(\mathfrak{g}) \otimes \operatorname{End}(V)+I(\tau) .
$$

Hence

$$
D_{1} D_{2}-\Gamma_{1}\left(D_{1}\right) D_{2} \in \mathfrak{n} U(\mathfrak{g}) \otimes \operatorname{End}(V)+I(\tau) D_{2} .
$$

Assume $D_{2}=\sum u_{i} \otimes T_{i}$, for $u_{i} \in U(\mathfrak{g})$, and $T_{i} \in \operatorname{End}(V)$. Then for any $X \in \mathfrak{k}$,

$$
\begin{aligned}
(X \otimes 1+1 \otimes \tau(X)) D_{2}= & \sum\left(X u_{i} \otimes T_{i}+u_{i} \otimes \tau(X) T_{i}\right) \\
= & \sum\left(\operatorname{ad}(X) u_{i} \otimes T_{i}+u_{i} \otimes\left[\tau(X), T_{i}\right]\right) \\
& +\sum\left(u_{i} X \otimes T_{i}+u_{i} \otimes T_{i} \tau(X)\right) .
\end{aligned}
$$

Then first summation is zero since $D_{2} \in(U(\mathfrak{g}) \otimes \operatorname{End}(V))^{K}$. The second one is just $D_{2}(X \otimes 1+1 \otimes \tau(X))$. So we have proved $I(\tau) D_{2} \subset I(\tau)$. Hence

$$
D_{1} D_{2}-\Gamma_{1}\left(D_{1}\right) D_{2} \in \mathfrak{n} U(\mathfrak{g}) \otimes \operatorname{End}(V)+I(\tau) .
$$


However,

$$
D_{2}-\Gamma_{1}\left(D_{2}\right) \in \mathfrak{n} U(\mathfrak{g}) \otimes \operatorname{End}(V)+I(\tau),
$$

and

$$
\Gamma_{1}\left(D_{1}\right)(\mathfrak{n} U(\mathfrak{g}) \otimes \operatorname{End}(V)+I(\tau)) \subset \mathfrak{n} U(\mathfrak{g}) \otimes \operatorname{End}(V)+I(\tau) .
$$

Therefore

$$
D_{1} D_{2}-\Gamma_{1}\left(D_{1}\right) \Gamma_{1}\left(D_{2}\right) \in \mathfrak{n} U(\mathfrak{g}) \otimes \operatorname{End}(V)+I(\tau) .
$$

This proves $\Gamma_{1}\left(D_{1} D_{2}\right)=\Gamma_{1}\left(D_{1}\right) \Gamma_{1}\left(D_{2}\right)$.

For $D=\sum u_{i} \otimes T_{i} \in U(\mathfrak{g}) \otimes \operatorname{End}(V)$, and $f \in C^{\infty}(G, V)$, we define

$$
\mu_{1}(D) f=\sum T_{i} R_{u_{i}} f .
$$

It is not difficult to show for $D \in(U(\mathfrak{g}) \otimes \operatorname{End}(V))^{K}$ and $f \in C^{\infty} \operatorname{Ind}_{K}^{G}(\tau), \mu_{1}(D) f$ remains in $C^{\infty} \operatorname{Ind}_{K}^{G}(\tau)$. So $\mu_{1}(D) \in D_{\tau}$. In fact $\mu_{1}$ is a surjective homomorphism from $(U(\mathfrak{g}) \otimes \operatorname{End}(V))^{K}$ onto $D_{\tau}$.

We define $\mu(D)=\mu_{1}(D \otimes 1)$, for each $D \in U(\mathfrak{g})^{K}$. By a theorem of Burnside which asserts that $\tau(U(\mathfrak{k}))=\operatorname{End}(V)$, one can prove $\mu$ is a surjective homomorphism from $U(\mathfrak{g})^{K}$ onto $D_{\tau}$, using the surjectivity of $\mu_{1}$.

For each $\lambda \in \mathfrak{a}_{\mathbb{C}}^{*}$, we introduce an important function $\Psi_{\lambda}$ on $G$ with values in $\operatorname{End}(V)$ as follows:

$$
\Psi_{\lambda}(n a k)=a^{\lambda+\rho} \tau(k)^{-1}
$$

for $n \in N, a \in A$, and $k \in K$. Here $\rho$ is the half sum of the positive roots for $(\mathfrak{g}, \mathfrak{a})$. Notice that for each $v \in V$, the function: $g \rightarrow \Psi_{\lambda}(g) \cdot v$ belongs to $C^{\infty} \operatorname{Ind}_{K}^{G}(\tau)$.

Proposition 1.3. For each $D \in U(\mathfrak{g})^{K}$, and $v \in V$,

$$
\mu(D)\left(\Psi_{\lambda} \cdot v\right)=\Psi_{\lambda} \cdot\left(\Gamma_{1}(D \otimes 1)(\lambda+\rho) v\right) .
$$

Proof. Since both sides are left $N$-invariant and behave in the same way under the right $K$-action, it is sufficient to show they are equal when restricted to $A$. By definition

$$
D \otimes 1=D_{1}+\Gamma_{1}(D \otimes 1)+D_{2},
$$

where $D_{1} \in \mathfrak{n} U(\mathfrak{g}) \otimes \operatorname{End}(V)$, and $D_{2} \in I(\tau)$. It is easy to see that

$$
\mu_{1}\left(D_{1}\right)\left(\Psi_{\lambda} \cdot v\right) \mid A=0,
$$

and

$$
\mu_{1}\left(D_{2}\right)\left(\Psi_{\lambda} \cdot v\right)=0
$$

So

$$
\mu(D)\left(\Psi_{\lambda} \cdot v\right) \mid A=a^{\lambda+\rho} \Gamma_{1}(D \otimes 1)(\lambda+\rho) v .
$$

Corollary 1.4. There exists a homomorphism $\Gamma^{\prime}: D_{\tau} \rightarrow U(\mathfrak{a}) \otimes \operatorname{End}_{M}(V)$. Moreover, for each $D \in U(\mathfrak{g})^{K}, \Gamma^{\prime}(\mu(D))=\Gamma_{1}(D \otimes 1)$.

Remark 1.5. It has been proved in Section 3 in [Min] that $\Gamma^{\prime}$ is injective, using results from [Lep]. 
In general $D_{\tau}$ is very complicated. For instance it is not abelian in most of the cases. For this reason we replace it by $\mu(\mathcal{Z}(\mathfrak{g}))$ which we denote by $Z_{\tau}$.

Choose $\mathfrak{t}$ a maximal abelian subalgebra in $\mathfrak{m}$. Then $\mathfrak{h}_{\mathbb{C}}=(\mathfrak{t}+\mathfrak{a})_{\mathbb{C}}$ is a Cartan subalgebra of $\mathfrak{g}_{\mathbb{C}}$. Let $\Sigma\left(\mathfrak{g}_{\mathbb{C}}, \mathfrak{h}_{\mathbb{C}}\right)$ the root system for $\left(\mathfrak{g}_{\mathbb{C}}, \mathfrak{h}_{\mathbb{C}}\right)$. Let $\Sigma^{+}\left(\mathfrak{g}_{\mathbb{C}}, \mathfrak{h}_{\mathbb{C}}\right)$ be the set of positive roots for some ordering, and $\mathfrak{g}_{\mathbb{C}}^{+}\left(\right.$resp. $\mathfrak{g}_{\mathbb{C}}^{-}$) the sum of positive (resp. negative) root spaces. Choose an ordering such that $\mathfrak{n} \subset \mathfrak{g}_{\mathbb{C}}^{+}$. We consider each $\lambda \in \mathfrak{a}_{\mathbb{C}}^{*}\left(\right.$ resp. $\left.\mathfrak{t}_{\mathbb{C}}^{*}\right)$ an element of $\mathfrak{h}_{\mathbb{C}}^{*}$ by the requirement that $\lambda$ be zero in $\mathfrak{t}$ (resp. $\mathfrak{a}$ ). Let

$$
P=\left\{\alpha \in \Sigma^{+}\left(\mathfrak{g}_{\mathbb{C}}, \mathfrak{h}_{\mathbb{C}}\right)|\alpha| \mathfrak{a} \neq 0\right\}, \quad P_{0}=\left\{\alpha \in \Sigma^{+}\left(\mathfrak{g}_{\mathbb{C}}, \mathfrak{h}_{\mathbb{C}}\right)|\alpha| \mathfrak{a}=0\right\}
$$

Write

$$
\rho=\frac{1}{2} \sum_{\alpha \in P} \alpha, \quad \rho_{0}=\frac{1}{2} \sum_{\alpha \in P_{0}} \alpha .
$$

Let $\Theta$ be the Cartan involution of $\mathfrak{g}$ with fixed point set $\mathfrak{k}$ and extend it to an automorphism of $\mathfrak{g}_{\mathbb{C}}$. Then $\alpha \rightarrow-\Theta \alpha$ is a permutation of $P$, so $\rho \mid \mathfrak{t}=0$. Hence $\rho$ can be viewed as the half sum of positive roots for $(\mathfrak{g}, \mathfrak{a})$.

Let $\gamma^{\prime}: \mathcal{Z}(\mathfrak{g}) \rightarrow U\left(\mathfrak{h}_{\mathbb{C}}\right)$ be defined by

$$
Z-\gamma^{\prime}(Z) \in \mathfrak{g}_{\mathbb{C}}^{-} U(\mathfrak{g})
$$

for $Z \in \mathcal{Z}(\mathfrak{g})$. We define $\gamma(Z)(\mu)=\gamma^{\prime}(Z)\left(\mu-\rho-\rho_{0}\right)$, for each $\mu \in \mathfrak{h}_{\mathbb{C}}^{*}$. This is the usual Harish-Chandra homomorphism.

Let $V=\bigoplus_{\sigma \in \widehat{M}} V(\sigma)$ be the decomposition into the $M$-isotypic parts. We say $\sigma \in \tau$ if $V(\sigma) \neq 0$.

For each irreducible representation $\left(\sigma, V_{\sigma}\right)$ of $M$, we get a Lie algebra representation of $\mathfrak{m}$ by differentiation. We denote the representation by $d \sigma$. In general this is not irreducible. Fortunately it is a multiple of an irreducible representation of $\mathfrak{m}$. This fact can be seen in the following way. Let $M_{0}$ be the identity component of $M$. By structure theory (see 1.1.3.8 in [War]) one can find $Z(A)$, a finite subgroup of $M$ where each element commutes with every element of $M_{0}$. Choose an irreducible representation $\left(\sigma, V_{1}\right)$ of $M_{0}$ in $\left(\sigma, V_{\sigma}\right)$. For each $z \in Z(A),\left(\sigma, \sigma(z) V_{1}\right)$ gives an irreducible representation of $M_{0}$ in $\left(\sigma, V_{\sigma}\right)$, which is equivalent to $\left(\sigma, V_{1}\right)$. Since $\sigma$ is irreducible, $V_{\sigma}=\sum_{z \in Z(A)} \sigma(z) V_{1}$. So by Schur's lemma the center $\mathcal{Z}(\mathfrak{m})$ of $U(\mathfrak{m})$ acts on $V_{\sigma}$ by scalars. The action is determined by $\Lambda_{\sigma} \in \sqrt{-1} \mathrm{t}^{*}$ as follows: For each $Z$ in $\mathcal{Z}(\mathfrak{m}), d \sigma(Z)=\gamma(Z)\left(\Lambda_{\sigma}\right) \mathrm{I}_{V_{\sigma}}$, where $\gamma$ is the Harish-Chandra homomorphism for $(\mathfrak{m}, \mathfrak{t})$, and $\mathrm{I}_{V_{\sigma}}$ the identity map of $V_{\sigma}$. We choose $\Lambda_{\sigma}$ the highest weight of $\sigma$ plus $\rho_{0}$.

Let $\Gamma: D_{\tau} \rightarrow U(\mathfrak{a}) \otimes \operatorname{End}_{M}(V)$ be defined by

$$
\Gamma(D)(\lambda)=\Gamma^{\prime}(D)(\lambda+\rho) .
$$

Theorem 1.6. For each $Z \in \mathcal{Z}(\mathfrak{g})$, and $\lambda \in \mathfrak{a}_{\mathbb{C}}^{*}$,

$$
\Gamma(\mu(Z))(\lambda) \mid V(\sigma)=\gamma(Z)\left(\lambda-\Lambda_{\sigma}\right) \mathrm{I}_{V(\sigma)} .
$$

We give a proof below using a well-known proposition about $\mathcal{Z}(\mathfrak{g})$. A more selfcontained proof is in [Wall]. First for the proof and later use we recall the definition of Poisson transforms.

Let $\left(\delta, V_{\delta}\right)$ be a finite dimensional representation of $B=M A N$, the minimal parabolic subgroup of $G$. Let $C^{\infty} \operatorname{Ind}_{B}^{G}(\delta)$ be the space defined by

$$
\left\{\phi \in C^{\infty}\left(G, V_{\delta}\right) \mid \phi(\text { gman })=a^{-\rho} \delta^{-1}(\operatorname{man}) \phi(g), \forall g \in G, \forall \operatorname{man} \in B\right\} .
$$


Let $C^{\infty} \operatorname{Ind}_{B}^{G}(\delta)$ be endowed with the topology from $C^{\infty}\left(G, V_{\delta}\right)$. We will specify the topology on $C^{\infty} \operatorname{Ind}_{K}^{G}(\tau)$ in the next section.

Definition 1.7. A Poisson transform is a continuous, linear, $G$-equivariant map from $C^{\infty} \operatorname{Ind}_{B}^{G}(\delta)$ into $C^{\infty} \operatorname{Ind}_{K}^{G}(\tau)$.

Given $T \in \operatorname{Hom}_{M}\left(V_{\delta}, V\right)$, and $\phi \in C^{\infty} \operatorname{Ind}_{B}^{G}(\delta)$, we write

$$
P_{T} \phi(g)=\int_{K} \tau(k) T(\phi(g k)) d k .
$$

One can easily check $P_{T}$ is a Poisson transform.

Proposition 1.8. The map $T \rightarrow P_{T}$ is a bijection from $\operatorname{Hom}_{M}\left(V_{\delta}, V\right)$ onto the space of Poisson transforms.

This result appears in [Ven]. We include a proof for completeness. Suppose $P$ is a Poisson transform from $C^{\infty} \operatorname{Ind}_{B}^{G}(\delta)$ into $C^{\infty} \operatorname{Ind}_{K}^{G}(\tau)$. Define the Poisson kernel $p \in\left[C^{\infty} \operatorname{Ind}_{B}^{G}(\delta)\right]^{\prime} \otimes V$, the strong topological dual of $C^{\infty} \operatorname{Ind}_{B}^{G}(\delta)$ tensored by $V$, by

$$
\langle p, \phi\rangle=(P \phi)(e),
$$

for each $\phi \in C^{\infty} \operatorname{Ind}_{B}^{G}(\delta)$. By the $G$-equivariance of $P$ the Poisson kernel completely determines $P$ by

$$
P \phi(x)=\left\langle p, L_{x^{-1}} \phi\right\rangle,
$$

for any $\phi \in C^{\infty} \operatorname{Ind}_{B}^{G}(\delta)$. Here $L_{x^{-1}} \phi(g)=\phi(x g)$.

By Section 9 there is a $K$-equivariant isomorphism between $\left(C^{\infty} \operatorname{Ind}_{B}^{G}(\delta)\right)^{\prime}$ and $C^{-\infty} \operatorname{Ind}_{M}^{K}(\check{\delta} \mid M)$, where $C^{-\infty} \operatorname{Ind}_{M}^{K}(\check{\delta} \mid M)$ denotes the space of vector-valued distributions $f: C^{\infty}(K, \mathbb{C}) \rightarrow V_{\delta}^{*}$, such that

$$
R_{m} f=\check{\delta}(m)^{-1} f,
$$

for any $m \in M$. Here $\check{\delta}$ is the dual representation of $\delta \mid M$. And $R_{m} f(\phi)=$ $f\left(R_{m^{-1}} \phi\right)$, with $\left(R_{m^{-1}} \phi\right)(k)=\phi\left(k m^{-1}\right)$. So

$$
p \in C^{-\infty} \operatorname{Ind}_{M}^{K}(\check{\delta} \mid M) \otimes V .
$$

However, for $\phi \in C^{\infty} \operatorname{Ind}_{B}^{G}(\delta)$,

$$
\left\langle p, L_{k} \phi\right\rangle=P\left(L_{k} \phi\right)(e)=P \phi\left(k^{-1}\right)=\tau(k)(P \phi(e))=\tau(k)(\langle p, \phi\rangle) .
$$

Hence $p \in\left(C^{-\infty} \operatorname{Ind}_{M}^{K}(\check{\delta} \mid M) \otimes V\right)^{K}$. Let $\pi$ be the representation of $K$ in $V_{\delta}^{*} \otimes$ $V$ defined by $\pi(k)(v \otimes w)=v \otimes \tau(k) w$, for $v \in V_{\delta}^{*}$, and $w \in V$. Then $p \in$ $C^{-\infty}\left(K, V_{\delta}^{*} \otimes V\right)$, and $L_{k} p=\pi\left(k^{-1}\right) p$. By Lemma $9.3 p$ must be smooth. Its transformation properties imply that $p$ is determined by $p(e)$, which belongs to $\left(V_{\delta}^{*} \otimes V\right)_{M} \cong \operatorname{Hom}_{M}\left(V_{\delta}, V\right)$.

Proof of Proposition 1.8. From the definition of $P_{T}$, it is immediate that the Poisson kernel of $P_{T}$ evaluated at the identity is $T$. This shows the map $T \rightarrow P_{T}$ is injective. On the other hand, let $P$ be a Poisson transform, and let $p$ be its Poisson 
kernel. Then

$$
\begin{aligned}
P \phi(x) & =\left\langle p, L_{x^{-1}} \phi\right\rangle \\
& =\int_{K}\langle p(k), \phi(x k)\rangle d k \\
& =\int_{K} \tau(k) p(e) \phi(x k) d k .
\end{aligned}
$$

This proves $P=P_{p(e)}$, whence the surjectivity.

\section{Lemma 1.9.}

$$
\int_{K} F\left(\mathrm{k}\left(g^{-1} k\right)\right) d k=\int_{K} F(k) e^{-2 \rho \mathrm{H}(g k)} d k .
$$

Let $\sigma$ be a finite dimensional representation of $M$ and $\lambda \in \mathfrak{a}_{\mathbb{C}}^{*}$. Then $\sigma \otimes(-\lambda) \otimes 1$ defines a representation of $B$ by $\operatorname{man} \rightarrow a^{-\lambda} \sigma(m)$.

Corollary 1.10. For each $\phi \in C^{\infty} \operatorname{Ind}_{B}^{G}(\sigma \otimes(-\lambda) \otimes 1), T \in \operatorname{Hom}_{M}\left(V_{\sigma}, V\right)$,

$$
P_{T} \phi(g)=\int_{K} \Psi_{\lambda}\left(k^{-1} g\right) T \phi(k) d k .
$$

Proof.

$$
\begin{aligned}
P_{T} \phi(g) & =\int_{K} \tau(k) T \phi(g k) d k \\
& =\int_{K} \tau(k) T \phi(\mathrm{k}(g k) \exp \mathrm{H}(g k) \mathrm{n}(g k)) d k \\
& =\int_{K} e^{(\lambda-\rho) \mathrm{H}(g k)} \tau(k) T \phi(\mathrm{k}(g k)) d k .
\end{aligned}
$$

By Lemma 1.9,

$$
\begin{aligned}
\int_{K} e^{(\lambda-\rho) \mathrm{H}(g k)} \tau(k) T \phi(\mathrm{k}(g k)) d k \\
\quad=\int_{K} e^{(\lambda+\rho) \mathrm{H}\left(g \mathrm{k}\left(g^{-1} k\right)\right)} \tau\left(\mathrm{k}\left(g^{-1} k\right)\right) T \phi\left(\mathrm{k}\left(g \mathrm{k}\left(g^{-1} k\right)\right)\right) d k \\
=\int_{K} e^{(-\lambda+\rho) \mathrm{H}\left(g^{-1} k\right)} \tau\left(\mathrm{k}\left(g^{-1} k\right)\right) T \phi(k) d k \\
=\int_{K} \Psi_{\lambda}\left(k^{-1} g\right) T \phi(k) d k .
\end{aligned}
$$

Proof of Theorem 1.6. Let $\delta$ be the restriction of $\tau$ to $M$ with $V(\sigma)$ as the representation space. It is well-known that $L_{Z} \phi=\gamma(Z)\left(\Lambda_{\sigma}-\lambda\right) \phi$ for each $Z \in \mathcal{Z}(\mathfrak{g})$, and $\phi \in C^{\infty} \operatorname{Ind}_{B}^{G}(\delta \otimes(-\lambda) \otimes 1)$. See [Vogan]. Let $*$ denote adjoint on $U(\mathfrak{g})$. By Corollary 5.31 on p. 324 in [Helg1],

$$
\begin{aligned}
R_{Z} P_{T} \phi & =L_{Z^{*}} P_{T} \phi=P_{T} L_{Z^{*}} \phi \\
& =P_{T}\left(\gamma\left(Z^{*}\right)\left(\Lambda_{\sigma}-\lambda\right) \phi\right)=P_{\gamma\left(Z^{*}\right)\left(\Lambda_{\sigma}-\lambda\right) T} \phi=P_{\gamma(Z)\left(-\Lambda_{\sigma}+\lambda\right) T} \phi .
\end{aligned}
$$

On the other hand, by Proposition 1.3 and Corollary 1.10,

$$
R_{Z} P_{T} \phi=P_{\Gamma(\mu(Z))(\lambda) T} \phi .
$$

So

$$
P_{\gamma(Z)\left(-\Lambda_{\sigma}+\lambda\right) T}=P_{\Gamma(\mu(Z))(\lambda) T}
$$


By Proposition 1.8 we conclude

$$
\Gamma(\mu(Z))(\lambda) \mid V(\sigma)=\gamma(Z)\left(\lambda-\Lambda_{\sigma}\right) \mathrm{I}_{V(\sigma)} .
$$

By definition a character of $Z_{\tau}$ is a homomorphism from $Z_{\tau}$ to $\mathbb{C}$.

Proposition 1.11. A character $\chi$ of $Z_{\tau}$ is given by $\lambda-\Lambda_{\sigma}$, where $\lambda \in \mathfrak{a}_{\mathbb{C}}^{*}$, and $\sigma \in \tau$. More specifically, $\chi(\mu(Z))=\gamma(Z)\left(\lambda-\Lambda_{\sigma}\right)$, for each $Z \in \mathcal{Z}(\mathfrak{g})$.

Lemma 1.12. Let $S$ be the common zeros of $p_{1}, \ldots, p_{m}$ in $S\left(\mathfrak{h}_{\mathbb{C}}\right)$. Assume in addition $S$ is $\widetilde{W}$ invariant, $\widetilde{W}$ denoting the Weyl group for $\left(\mathfrak{g}_{\mathbb{C}}, \mathfrak{h}_{\mathbb{C}}\right)$. Then one can find $q_{1}, \ldots, q_{n}$ in $I\left(\mathfrak{h}_{\mathbb{C}}\right)$, the $\widetilde{W}$-invariants in $S\left(\mathfrak{h}_{\mathbb{C}}\right)$, such that $S$ is the common zeros of $q_{1}, \ldots, q_{n}$.

Proof. Write $R_{i}(X)=\prod_{s \in \widetilde{W}}\left(X-p_{i}^{s}\right)$, and $w=|\widetilde{W}|$. Then

$$
R_{i}(X)=X^{w}+p_{i 1} X^{w-1}+\cdots+p_{i w} .
$$

It is easy to see we can use $p_{i j}$ as our $q_{1}, \ldots, q_{n}$.

Proof of Proposition 1.11. Let $A=\mu \circ \gamma^{-1}: I\left(\mathfrak{h}_{\mathbb{C}}\right) \rightarrow Z_{\tau}$. By Theorem $1.6 \operatorname{ker}(A)=$ $\left\{\rho \in I\left(\mathfrak{h}_{\mathbb{C}}\right)|p|\left(-\Lambda_{\sigma}+\mathfrak{a}_{\mathbb{C}}^{*}\right)=0\right.$, for all $\left.\sigma \in \tau\right\}$. Here we use Remark 1.5 which asserts that $\Gamma$ is injective. Suppose $\chi: Z_{\tau} \rightarrow \mathbb{C}$ is a character of $Z_{\tau}$. Then there exists $\mu \in \mathfrak{h}_{\mathbb{C}}^{*}$, such that $\chi \circ A=\chi_{\mu}$, where $\chi_{\mu}$ is the homomorphism defined by evaluation at $\mu$. Obviously $p(\mu)=0$, for all $p \in \operatorname{ker}(A)$. Let

$$
S=\bigcup_{\sigma \in \tau, w \in \widetilde{W}} w\left(-\Lambda_{\sigma}+\mathfrak{a}_{\mathbb{C}}^{*}\right) \subset \mathfrak{h}_{\mathbb{C}}^{*}
$$

Obviously one can find $p_{1}, \ldots, p_{m}$ in $S\left(\mathfrak{h}_{\mathbb{C}}\right)$ such that $S$ is the common zeros of $p_{1}, \ldots, p_{m}$. Then by Lemma 1.12 we can find $q_{1}, \ldots, q_{n}$ in $I\left(\mathfrak{h}_{\mathbb{C}}\right)$ such that $S$ is the common zeros of $q_{1}, \ldots, q_{n}$. This shows $q_{1}, \ldots, q_{n}$ are in $\operatorname{ker}(A)$. So $q_{1}(\mu)=\cdots=$ $q_{n}(\mu)$. Therefore $\mu \in S$, i.e. $\mu=w\left(\lambda-\Lambda_{\sigma}\right)$ for some $\lambda \in \mathfrak{a}_{\mathbb{C}}^{*}, \sigma \in \tau$, and $w \in \widetilde{W}$.

For $s \in M^{\prime}$, define $s .(X \otimes T)=\operatorname{Ad}(s) X \otimes \tau(s) T \tau\left(s^{-1}\right)$, for $X \in U(\mathfrak{a})$, and $T \in \operatorname{End}(V)$. The next proposition is about a property of the generalized HarishChandra homomorphism. It is a weak version of a conjecture by Lepowsky.

Proposition 1.13. For each $s \in M^{\prime} / M$, s. $\Gamma(D)=\Gamma(D)$, for each $D \in Z_{\tau}$.

For the proof of this result we need more facts about Weyl groups. Let $\widetilde{W}_{1} \subset \widetilde{W}$ be the subgroup where every element stabilizes $\mathfrak{a}$. It is well-known there is a surjective homomorphism $\widetilde{W}_{1} \rightarrow M^{\prime} / M$. The kernel $\widetilde{W}_{0}$ is the Weyl group for $(\mathfrak{m}, \mathfrak{t})$.

Lemma 1.14. For each $s \in M^{\prime} / M$, choose $w(s)$ in $\widetilde{W}_{1}$ in the preimage of $s$ under the homomorphism above. Then $\Lambda_{\sigma^{s}}=w(s) \Lambda_{\sigma}$.

Proof (by Vogan). Take a maximal torus $T$ of $M_{0} . s T s^{-1}$ is another maximal torus. So there is $m \in M_{0}$, such that $m s T s^{-1} m^{-1}=T$. To avoid cumbersome notations we assume $s T s^{-1}=T$. It is easy to see that $\operatorname{Ad}(s)^{*}$, the transpose of $\operatorname{Ad}(s)$, preserves $\Sigma(\mathfrak{m}, \mathfrak{t})$. We can also assume $\operatorname{Ad}(s)^{*}$ preserves $\Sigma^{+}(\mathfrak{m}, \mathfrak{t})$. For $Z \in \mathcal{Z}(\mathfrak{m})$,

$$
Z-\gamma^{\prime}(Z) \in \mathfrak{m}^{-} U(\mathfrak{m})
$$


Hence

$$
\operatorname{Ad}(s) Z-\operatorname{Ad}(s) \gamma^{\prime}(Z) \in \mathfrak{m}^{-} U(\mathfrak{m})
$$

So

$$
\begin{aligned}
\sigma^{s}(Z) & =\sigma(\operatorname{Ad}(s) Z)=\operatorname{Ad}(s) \gamma^{\prime}(Z)\left(\Lambda_{\sigma}-\rho_{0}\right) \\
& =\gamma^{\prime}(Z)\left(\operatorname{Ad}(s)^{*} \Lambda_{\sigma}-\rho_{0}\right)=\gamma(Z)\left(\operatorname{Ad}(s)^{*} \Lambda_{\sigma}\right) .
\end{aligned}
$$

Hence

$$
\Lambda_{\sigma^{s}}=\operatorname{Ad}(s)^{*} \Lambda_{\sigma}=w(s) \Lambda_{\sigma} .
$$

Proof of Proposition 1.13. Take $Z \in \mathcal{Z}(\mathfrak{g})$ such that $D=\mu(Z)$. Then for each $\lambda \in \mathfrak{a}_{\mathbb{C}}^{*}$, and $s \in M^{\prime}$,

$$
s . \Gamma(D)(\lambda)|V(\sigma)=s . \Gamma(\mu(Z))(\lambda)| V(\sigma)=\gamma(Z)\left(\operatorname{Ad}(s)^{*} \lambda-\Lambda_{\sigma^{s}}\right) \mathrm{I}_{V(\sigma)} .
$$

By Lemma 1.14, $\Lambda_{\sigma^{s}}=w(s) \Lambda_{\sigma}$. So

$$
\begin{aligned}
s . \Gamma(D)(\lambda) \mid V(\sigma) & =\gamma(Z)\left(\operatorname{Ad}(s)^{*} \lambda-w(s) \Lambda_{\sigma}\right) \mathrm{I}_{V(\sigma)} \\
& =\gamma(Z)\left(\lambda-\Lambda_{\sigma}\right) \mathrm{I}_{V(\sigma)} \\
& =\Gamma(\mu(Z))(\lambda) \mid V(\sigma) \\
& =\Gamma(D)(\lambda) \mid V(\sigma) .
\end{aligned}
$$

Now let $\overline{\mathfrak{n}}=\theta \mathfrak{n}$. Similarly as in Proposition 1.1 we get

$$
U(\mathfrak{g}) \otimes \operatorname{End}(V)=U(\mathfrak{a}) \otimes \operatorname{End}(V) \oplus(\overline{\mathfrak{n}} U(\mathfrak{g}) \otimes \operatorname{End}(V)+I(\tau)) .
$$

Then we define $\widetilde{\Gamma}_{1}: U(\mathfrak{g}) \otimes \operatorname{End}(V) \rightarrow U(\mathfrak{a}) \otimes \operatorname{End}(V)$ as the projection according to this decomposition.

Corollary 1.15. For each $Z \in \mathcal{Z}(\mathfrak{g})$, and $\lambda \in \mathfrak{a}_{\mathbb{C}}^{*}$,

$$
\widetilde{\Gamma}_{1}(Z \otimes 1)(\lambda)=\Gamma(\mu(Z))(\lambda+\rho) .
$$

Proof. Take $s \in M^{\prime}$ such that $\operatorname{Ad}(s)^{*} \Sigma^{+}(\mathfrak{g}, \mathfrak{a})=\Sigma^{-}(\mathfrak{g}, \mathfrak{a})$. By definition

$$
Z \otimes 1-\Gamma_{1}(Z \otimes 1) \in \mathfrak{n} U(\mathfrak{g}) \otimes \operatorname{End}(V)+I(\tau) .
$$

Hence

$$
s .(Z \otimes 1)-s . \Gamma_{1}(Z \otimes 1) \in \overline{\mathfrak{n}} U(\mathfrak{g}) \otimes \operatorname{End}(V)+I(\tau) .
$$

So

$$
\widetilde{\Gamma}_{1}(Z \otimes 1)=s \cdot \Gamma_{1}(Z \otimes 1) .
$$

Hence

$$
\begin{aligned}
\widetilde{\Gamma}_{1}(Z \otimes 1)(\lambda) & =\tau(s) \Gamma_{1}(Z \otimes 1)\left(\operatorname{Ad}(s)^{*} \lambda\right) \tau\left(s^{-1}\right) \\
& =\tau(s) \Gamma(\mu(Z))\left(\operatorname{Ad}(s)^{*} \lambda-\rho\right) \tau\left(s^{-1}\right) \\
& =\tau(s) \Gamma(\mu(Z))\left(\operatorname{Ad}(s)^{*}(\lambda+\rho)\right) \tau\left(s^{-1}\right) \\
& =\Gamma(\mu(Z))(\lambda+\rho) . \quad \square
\end{aligned}
$$




\section{SOME FUNCTION SPACES ON $G$}

In this section we introduce a certain growth condition on a function on $G$ with values in $V$. It turns out the condition is satisfied by $P_{T} \phi$ for any $\phi \in C^{\infty} \operatorname{Ind}_{B}^{G}(\delta)$, where $\delta$ is a certain finite dimensional representation of $B$.

For each $g \in G$, we denote by $\|g\|$ the operator norm of $\operatorname{Ad}(g)$ on $\mathfrak{g}$, which is equipped with the inner product $\langle X, Y\rangle_{\Theta}=-K(X, \Theta Y)$. Here $K$ is the Killing form on $\mathfrak{g}$.

Lemma 2.1. (i) $\|g\|=\|\Theta g\|=\left\|g^{-1}\right\| \geq 1$;

(ii) $\left\|g_{1} g_{2}\right\| \leq\left\|g_{1}\right\|\left\|g_{2}\right\|$;

(iii) if $g=k_{1} a k_{2}$ with $k_{1}, k_{2} \in K, a \in A$, then

$$
\|g\|=\exp \left(\max _{\alpha \in \Sigma(\mathfrak{g}, \mathfrak{a})}|\alpha(\log a)|\right) ;
$$

(iv) there are constants $C_{1}, C_{2}>0$, such that if $x=\exp X$ with $X \in \mathfrak{p}$, then $e^{C_{1}|X|} \leq\|x\| \leq e^{C_{2}|X|}$. Here $\mathfrak{p}$ is the -1 eigenspace of $\Theta$, and $|X|=\sqrt{\langle X, X\rangle_{\Theta}}$;

(v) $\|a\| \leq \|$ an $\|$, for $a \in A$, and $n \in N$.

Proof. See $[\mathrm{BS}]$.

For any function $f: G \rightarrow V$ and $r \in \mathbb{R}$, we write

$$
\|f\|_{r}=\sup _{g \in G}\|g\|^{-r}|f(g)| .
$$

We say $f$ increases at most exponentially if $\|f\|_{r}<\infty$, for some $r \in \mathbb{R}$. Let $C_{r}(G, V)$ denote the Banach space of continuous functions $f$ on $G$ with values in $V$ with $\|f\|_{r} \leq \infty$.

Example 2.2. Let $\lambda \in \mathfrak{a}_{\mathbb{C}}^{*}$, and $\sigma$ a finite dimensional representation of $M$. Let $r(\lambda)=C_{1}^{-1}|\operatorname{Re} \lambda-\rho|$, where $C_{1}$ is the constant in Lemma 2.1 (iv). Then for each $\phi \in C^{\infty} \operatorname{Ind}_{B}^{G}(\sigma \otimes(-\lambda) \otimes 1)$, and $T \in \operatorname{Hom}_{M}\left(V_{\sigma}, V\right), P_{T} \phi$ belongs to $C_{r(\lambda)}(G, V)$. This is in [BS] when $\tau$ is trivial and $\tau$ in general does not offer additional difficulties.

Write

$$
C_{r}^{\infty}(G, V)=\left\{f \in C^{\infty}(G, V) \mid L_{u} f \in C_{r}(G, V), \forall u \in U(\mathfrak{g})\right\} .
$$

We endow $C_{r}(G, V)$ with its standard topology: Let $X_{1}, \ldots, X_{p}$ be a basis of $\mathfrak{g}$, and $X^{I}=X^{i_{1}} \cdots X^{i_{p}} \in U(\mathfrak{g})$ for $I=\left(i_{1}, \ldots, i_{p}\right) \in \mathbb{N}^{p}$. For $q \in \mathbb{N}$ and $f \in C^{q}(G, V)$, a $q$ times continuously differentiable function from $G$ to $V$, we define

$$
\|f\|_{q, r}=\sum_{|I| \leq q}\left\|L_{X^{I}} f\right\|_{r} .
$$

Endowed with this norm the space

$$
C_{r}^{q}(G, V)=\left\{f \in C^{q}(G, V)\|\| f \|_{q, r}<\infty\right\}
$$

is a Banach space. Obviously $C_{r}^{q} \subset C_{r}^{q^{\prime}}$ if $q^{\prime} \leq q, C_{r}^{\infty}(G, V)=\bigcap_{q} C_{r}^{q}(G, V)$. The topology on $C_{r}^{\infty}(G, V)$ is given by the family of norms $\|\cdot\|_{q, r}, q \in \mathbb{N}$, on $C_{r}^{\infty}(G, V)$. We now consider for each $q \in \mathbb{N}$ the action of $L$ and $R$ on $C_{r}^{\infty}(G, V)$. Recall for $g, x \in G$, and $f \in C^{q}(G, V), L_{x} f(g)=f\left(x^{-1} g\right)$, and $R_{x} f(g)=f(g x)$. Obviously $L_{x}$ leaves $C_{r}^{q}(G, V)$ invariant. In fact $\left\|L_{x} f\right\|_{q, r} \leq C\|x\|^{r+s}\|f\|_{q, r}$, for each $f \in C_{r}^{q}(G, V)$, and $x \in G$. Here $C$ and $s$ are constants. On the other hand, 
$\left\|R_{x} f\right\|_{q, r} \leq\|x\|^{r}\|f\|_{q, r}$. From Example 2.2, we see $P_{T}$ maps $C^{\infty} \operatorname{Ind}_{B}^{G}(\sigma \otimes(-\lambda) \otimes 1)$ into $C_{r(\lambda)}^{\infty}(G, V)$ continuously.

Recall from Proposition 1.11 a character of $Z_{\tau}$ is given by $\lambda-\Lambda$, where $\lambda \in \mathfrak{a}_{\mathbb{C}}^{*}$, and $\Lambda$ is the infinitesimal character of an irreducible representation of $M$ in $\tau$. Let $\mathcal{E}_{\lambda-\Lambda} \operatorname{Ind}_{K}^{G}(\tau)$ denote the corresponding eigenspace of $Z_{\tau}$. Let

$$
\begin{aligned}
\mathcal{E}_{\lambda-\Lambda, r}^{\infty} \operatorname{Ind}_{K}^{G}(\tau) & =\mathcal{E}_{\lambda-\Lambda} \operatorname{Ind}_{K}^{G}(\tau) \cap C_{r}^{\infty}(G, V), \\
\mathcal{E}_{\lambda-\Lambda}^{\infty} \operatorname{Ind}_{K}^{G}(\tau) & =\bigcup_{r \in \mathbb{R}} \mathcal{E}_{\lambda-\Lambda, r}^{\infty} \operatorname{Ind}_{K}^{G}(\tau) .
\end{aligned}
$$

Our goal is to describe $\mathcal{E}_{\lambda-\Lambda}^{\infty} \operatorname{Ind}_{K}^{G}(\tau)$ in terms of the Poisson transform, at least for "generic" $\lambda-\Lambda$. The following result due to Harish-Chandra is very important to us. See [Wall2].

Proposition 2.3. Let $\bar{N}=\Theta N$. Then $C(\lambda)$ defined by

$$
C(\lambda)=\int_{\bar{N}} \tau(\mathrm{k}(\bar{n})) e^{-(\lambda+\rho) \mathrm{H}(\bar{n})} d \bar{n}
$$

is holomorphic on $\left\{\lambda \in \mathfrak{a}_{\mathbb{C}}^{*} \mid \operatorname{Re}\langle\lambda, \alpha\rangle>0\right.$, for each $\left.\alpha \in \Sigma^{+}(\mathfrak{g}, \mathfrak{a})\right\}$. Moreover there exists a meromorphic continuation to $\mathfrak{a}_{\mathbb{C}}^{*}$.

Proposition 2.4. Let $\lambda \in \mathfrak{a}_{\mathbb{C}}^{*}$ such that $\operatorname{Re}\langle\lambda, \alpha\rangle>0$, for $\alpha \in \Sigma^{+}(\mathfrak{g}, \mathfrak{a})$. Then

$$
\lim _{t \rightarrow \infty} e^{(-\lambda+\rho)(H)} P_{T} \phi(g \exp t H)=C(\lambda) T \phi,
$$

for each $H \in \mathfrak{a}^{+}, T \in \operatorname{Hom}_{M}\left(V_{\sigma}, V\right)$, and $\phi \in C^{\infty} \operatorname{Ind}_{B}^{G}(\sigma \otimes(-\lambda) \otimes 1)$. Here $\mathfrak{a}^{+}=\left\{X \in \mathfrak{a} \mid \alpha(X)>0, \forall \alpha \in \Sigma^{+}(\mathfrak{g}, \mathfrak{a})\right\}$.

Proof. First we observe $k \rightarrow \tau(k) T \phi(g \exp t H k)$ is a function on $K / M$. By Theorem 5.20 in Chapter I in [Helg1],

$$
\begin{aligned}
P_{T} \phi(g \exp t H) & =\int_{\bar{N}} \tau(\mathrm{k}(\bar{n})) T \phi(g \exp t H \mathrm{k}(\bar{n})) e^{-2 \rho \mathrm{H}(\bar{n})} d \bar{n} \\
& =\int_{\bar{N}} e^{-(\lambda+\rho) \mathrm{H}(\bar{n})} \tau(\mathrm{k}(\bar{n})) T \phi(g \exp t H \bar{n}) d \bar{n} \\
& =e^{(\lambda-\rho) t H} \int_{\bar{N}} e^{-(\lambda+\rho) \mathrm{H}(\bar{n})} \tau(\mathrm{k}(\bar{n})) T \phi\left(g a_{t} \bar{n} a_{t}^{-1}\right) d \bar{n} .
\end{aligned}
$$

Here $a_{t}=\exp t H$. So

$$
e^{-(\lambda-\rho) t H} P_{T} \phi(g \exp t H)=\int_{\bar{N}} e^{-(\lambda+\rho) \mathrm{H}(\bar{n})} \tau(\mathrm{k}(\bar{n})) T \phi\left(g a_{t} \bar{n} a_{t}^{-1}\right) d \bar{n}
$$

since $a_{t} \bar{n} a_{t}^{-1} \rightarrow e$, as $t \rightarrow \infty$. Formally we have

$$
P_{T} \phi(g \exp t H) \rightarrow C(\lambda) T \phi(g),
$$

as $t \rightarrow \infty$. To justify the exchange of two limits we use an argument due to Helgason. Let $\lambda=\xi+\sqrt{-1} \eta$, for $\xi, \eta \in \mathfrak{a}^{*}$. Our assumption on $\lambda$ amounts to $A_{\xi} \in \mathfrak{a}^{+}$, where $A_{\xi}$ is given by $\left\langle\mu, A_{\xi}\right\rangle=K(\xi, \mu)$, for each $\mu \in \mathfrak{a}^{*}$. It was proved by Harish-Chandra that

$$
B(H, \mathrm{H}(\bar{n})) \geq 0, \quad B\left(H, \mathrm{H}(\bar{n})-\mathrm{H}\left(a_{t} \bar{n} a_{t}^{-1}\right)\right) \geq 0,
$$

for each $H \in \mathfrak{a}^{+}$. Thus if we choose $\varepsilon$ such that $0<\varepsilon<1, A_{\rho}-\varepsilon A_{\xi} \in \mathfrak{a}^{+}$, and put

$$
C=\sup _{\bar{n}, t}\left|\tau(\mathrm{k}(\bar{n})) T \phi\left(g \mathrm{k}\left(a_{t} \bar{n} a_{t}^{-1}\right)\right)\right|<\infty,
$$


then

$$
\begin{aligned}
\left|e^{-(\lambda+\rho) \mathrm{H}(\bar{n})} \tau(\mathrm{k}(\bar{n})) T \phi\left(g a_{t} \bar{n} a_{t}^{-1}\right)\right| \\
\quad=\left|e^{-(\lambda+\rho) \mathrm{H}(\bar{n})} e^{(\lambda-\rho) \mathrm{H}\left(a_{t} \bar{n} a_{t}^{-1}\right)} \tau(\mathrm{k}(\bar{n})) T \phi\left(g \mathrm{k}\left(a_{t} \bar{n} a_{t}^{-1}\right)\right)\right| \\
\quad \leq C e^{-(\xi+\rho) \mathrm{H}(\bar{n})} e^{(\xi-\rho) \mathrm{H}\left(a_{t} \bar{n} a_{t}^{-1}\right)} \\
\quad \leq C e^{-(\xi+\rho) \mathrm{H}(\bar{n})} e^{(\xi-\varepsilon \xi) \mathrm{H}\left(a_{t} \bar{n} a_{t}^{-1}\right)} \\
\quad \leq C e^{-(\xi+\rho) \mathrm{H}(\bar{n})} e^{(\xi-\varepsilon \xi) \mathrm{H}(\bar{n})} \\
\quad \leq C e^{(-\varepsilon \xi-\rho) \mathrm{H}(\bar{n})}
\end{aligned}
$$

This being integrable over $\bar{N}$ justifies letting $t \rightarrow \infty$ under the integral sign and proves Proposition 2.4.

\section{Asymptotics}

By a formal expansion at a point $H_{0} \in \mathfrak{a}^{+}$, we mean a formal sum

$$
\sum_{\xi \in X} p_{\xi}(H, t) e^{t \xi(H)}
$$

where $X$ is a subset of $\mathfrak{a}_{\mathbb{C}}^{*}$ such that the subset $X(N)$ given by

$$
X(N)=\left\{\xi \in X \mid \operatorname{Re} \xi\left(H_{0}\right) \geq N\right\}
$$

is a finite set for each $N \in \mathbb{R}$, where $p_{\xi}$ is a continuous function defined in a neighborhood of $\left\{H_{0}\right\} \times \mathbb{R}$ and polynomial in the last variable.

Let $f$ be a function $\mathfrak{a}^{+} \rightarrow V$. If $N \in \mathbb{R}$ we say the formal sum is asymptotic to $f$ of order $N$ at $H_{0}$, if there exist a neighborhood of $H_{0}$ in $\mathfrak{a}^{+}$, say $U$, and constants $\varepsilon \geq 0, C \geq 0$, such that

$$
\left|f(t H)-\sum_{\xi \in X(N)} p_{\xi}(H, t) e^{t \xi(H)}\right| \leq C e^{(N-\varepsilon) t},
$$

for each $H \in U, t \geq 0$. Moreover, we say the formal expansion is an asymptotic expansion for $f$ at $H_{0}$ if for every $N \in \mathbb{R}$ it is asymptotic to $f$ of order $N$ at $H_{0}$. We write this as

$$
f(t H) \sim \sum_{\xi \in X} p_{\xi}(H, t) e^{t \xi(H)} \quad(t \rightarrow \infty) .
$$

The following result shows that the $p_{\xi}$ 's are essentially unique.

Proposition 3.1. Let $X \subset \mathfrak{a}_{\mathbb{C}}^{*}, \sum_{\xi \in X} p_{\xi}(H, t) e^{t \xi(H)}$ and $\sum_{\xi \in X} q_{\xi}(H, t) e^{t \xi(H)}$ be formal expansions at $H_{0}$, both assumed to be asymptotic to $f: \mathfrak{a}^{+} \rightarrow V$. Then for each $\xi \in X$, there is a neighborhood $U$ of $H_{0}$, such that $p_{\xi}=q_{\xi}$ on $U \times \mathbb{R}$.

Proof. See Proposition 3.1 in [BS].

Let $\lambda-\Lambda$ be a character of $Z_{\tau}$ in the sense of Proposition 1.11, where $\lambda \in \mathfrak{a}_{\mathbb{C}}^{*}$, and $\Lambda$ is given by the infinitesimal character of an irreducible representation of $M$. Let $X(\lambda, \Lambda)$ be the subset of $\mathfrak{a}_{\mathbb{C}}^{*}$ defined by

$$
X(\lambda, \Lambda)=\left\{w(\lambda-\Lambda)+\Lambda_{\sigma}-\rho-\mathbb{N} \cdot \Delta\left|w \in \widetilde{W}, \sigma \in \tau,\left(w(\lambda-\Lambda)+\Lambda_{\sigma}\right)\right| \mathfrak{t}=0\right\} .
$$

Then we have the following results. 
Theorem 3.2. (i) For each $f \in \mathcal{E}_{\lambda-\Lambda}^{\infty} \operatorname{Ind}_{K}^{G}(\tau), x \in G$, and $\xi \in X(\lambda, \Lambda)$, there exists a unique polynomial $p_{\lambda, \xi}(f, x, \cdot)$ on $\mathfrak{a}$ with values in $V$, such that

$$
f(t H) \sim \sum_{\xi \in X(\lambda, \Lambda)} p_{\lambda, \xi}(f, x, t H) e^{t \xi(H)} \quad(t \rightarrow \infty),
$$

at every $H_{0} \in \mathfrak{a}^{+}$, and the polynomials have degree $\leq d$, where $d$ is the number of elements in $\Sigma^{+}\left(\mathfrak{g}_{\mathbb{C}}, \mathfrak{h}_{\mathbb{C}}\right)$.

(ii) Let $r \in \mathbb{R}$ and $\xi \in X(\lambda, \Lambda)$; there exists $r^{\prime} \in \mathbb{R}$ such that $f \rightarrow p_{\lambda, \xi}(f, \cdot, \cdot)$ is a continuous map of $\mathcal{E}_{\lambda-\Lambda, r}^{\infty} \operatorname{Ind}_{K}^{G}(\tau)$ into $C_{r^{\prime}}^{\infty}(G, V) \otimes P_{d}(\mathfrak{a})$, equivariant for the left action of $G$ on $\mathcal{E}_{\lambda-\Lambda, r}^{\infty} \operatorname{Ind}_{K}^{G}(\tau)$ to $C_{r^{\prime}}^{\infty}(G, V) \otimes P_{d}(\mathfrak{a})$.

Theorem 3.3. Let $\Omega$ be an open set in $\mathfrak{a}_{\mathbb{C}}^{*}$. Let $\left\{f_{\lambda}\right\}_{\lambda \in \Omega}$ be a holomorphic family in $C_{r}^{\infty} \operatorname{Ind}_{K}^{G}(\tau)$ such that $f_{\lambda} \in \mathcal{E}_{\lambda-\Lambda, r}^{\infty} \operatorname{Ind}_{K}^{G}(\tau)$ for each $\lambda \in \Omega$. Fix $\lambda_{0} \in \Omega$ and $\xi_{0} \in X\left(\lambda_{0}, \Lambda\right)$. Let

$$
\Xi(\lambda)=\left\{w(\lambda-\Lambda)+\Lambda_{\sigma}-\rho-\mu \in X(\lambda, \Lambda) \mid w\left(\lambda_{0}-\Lambda\right)+\Lambda_{\sigma}-\rho-\mu=\xi_{0}\right\} .
$$

There exist an open neighborhood $\Omega_{0} \subset \Omega$ of $\lambda_{0}$ and a constant $r^{\prime} \in \mathbb{R}$ such that the $\operatorname{map}(\lambda, H) \rightarrow \sum_{\xi \in \Xi(\lambda)} p_{\lambda, \xi}\left(f_{\lambda}, \cdot, H\right) e^{\xi(H)}$ is continuous from $\Omega \times \mathfrak{a}^{+}$into $C_{r^{\prime}}^{\infty}(G, V)$ and in addition holomorphic in $\lambda$.

We shall prove these results in Sections 5 and 6.

\section{Some algebraic Results}

This section is a necessary preparation for the proof of the theorems stated in last section. It is strongly influenced by [Ban] and [BS].

Let $E$ be the set of $W$-harmonic polynomials in $\mathfrak{a}^{*}$. It is well-known that $j: E \otimes$ $I(\mathfrak{a}) \rightarrow S(\mathfrak{a})$ is bijective, where $j(e \otimes h)=e h$.

Now let $r: I\left(\mathfrak{h}_{\mathbb{C}}\right) \rightarrow I(\mathfrak{a})$ be the restriction map. We assume $r$ is surjective for the rest of the thesis. According to [Helg3] if $G / K$ is irreducible there are just four exceptions, and they only occur among symmetric spaces of exceptional groups. Pick a set of algebraically independent homogeneous generators of $I(\mathfrak{a})$, say, $p_{1}, \ldots, p_{m}$. Choose homogeneous elements $q_{1}, \ldots, q_{m}$ in $I\left(\mathfrak{h}_{\mathbb{C}}\right)$, such that $r\left(q_{i}\right)=p_{i}$, for $i=1, \ldots, m$. Let $I_{1}\left(\mathfrak{h}_{\mathbb{C}}\right)$ be the polynomial ring of $q_{1}, \ldots, q_{m}$.

For any $\mu \in \mathfrak{h}_{\mathbb{C}}^{*}$, let

$$
I_{1, \mu}^{-}=\left\{\left(T_{\mu} p\right)^{-} \mid p \in I_{1}\left(\mathfrak{h}_{\mathbb{C}}\right)\right\} .
$$

Here $\left(T_{\mu} p\right)(\nu)=p(\mu+\nu)$, for each $\nu \in \mathfrak{h}_{\mathbb{C}}^{*}$, and $\left(T_{\mu} p\right)^{-}(\lambda)=p(\mu+\lambda)$, for each $\lambda \in \mathfrak{a}^{*}$.

Proposition 4.1. Let $j_{\mu}: E \otimes I_{1, \mu}^{-} \rightarrow S(\mathfrak{a})$ be defined by

$$
j_{\mu}(e \otimes h)=e h .
$$

Then $j_{\mu}$ is bijective.

Proof. Observe $\left(T_{\mu} q_{i}\right)^{-}=p_{i}+r_{i}$, with $\operatorname{deg} r_{i}<\operatorname{deg} p_{i}$. Using the fact that $j$ is bijective and by induction we are done.

Let $\mathcal{Z}_{1}(\mathfrak{g})=\gamma^{-1}\left(\mathrm{I}_{1}(\mathfrak{h})\right)$. Here $\gamma$ is the Harish-Chandra homomorphism. For each $\lambda \in \mathfrak{a}_{\mathbb{C}}^{*}, \Lambda=\Lambda_{\sigma}$ for some $\sigma \in \tau$, let

$$
I(\lambda, \Lambda)=\left\{Z \in \mathcal{Z}_{1}(\mathfrak{g}) \mid \gamma(Z)(\lambda-\Lambda)=0\right\} .
$$


Recall $I(\tau)$ is the left ideal of $U(\mathfrak{g}) \otimes \operatorname{End}(V)$ generated by $X \otimes 1+1 \otimes \tau(X)$, for all $X \in \mathfrak{k}$. Let $J(\lambda, \Lambda)$ be the left ideal generated by $I(\lambda, \Lambda)$ and $I(\tau)$. Let

$$
\mathfrak{Y}_{\lambda, \Lambda}=U(\mathfrak{g}) \otimes \operatorname{End}(V) / J(\lambda, \Lambda) .
$$

Our interest in $\mathfrak{Y}_{\lambda, \Lambda}$ comes from the fact that for $f \in \mathcal{E}_{\lambda-\Lambda} \operatorname{Ind}_{K}^{G}(\tau)$, the map $u \otimes T \rightarrow T R_{u} f$ factors through $\mathfrak{Y}_{\lambda, \Lambda}$ since $f$ is killed by $J(\lambda, \Lambda)$. We shall find below an underlying vector space for $\mathfrak{Y}_{\lambda, \Lambda}$ independent of $\lambda$.

Write $\mathfrak{Y}=U(\overline{\mathfrak{n}}) \otimes E \otimes \operatorname{End}(V)$. We shall construct a linear bijection of $\mathfrak{Y}$ with $\mathfrak{Y}_{\lambda, \Lambda}$. First we identify $\mathfrak{Y}$ with a subspace of $U(\mathfrak{g}) \otimes \operatorname{End}(V)$ as follows: $u \otimes e \otimes T \rightarrow(u \cdot e) \otimes T$, for $u \in U(\overline{\mathfrak{n}}), e \in E$, and $T \in \operatorname{End}(V)$. Here $\cdot$ denotes the multiplication in $U(\mathfrak{a}+\overline{\mathfrak{n}})$. Let $\Psi: \mathfrak{Y} \otimes \mathcal{Z}_{1}(\mathfrak{g}) \rightarrow U(\mathfrak{g}) \otimes \operatorname{End}(V) / I(\tau)$ be the map defined by

$$
\Psi(y \otimes Z)=y \cdot(Z \otimes 1)+I(\tau),
$$

for $y \in \mathfrak{Y}, Z \in \mathcal{Z}_{1}(\mathfrak{g})$. Here $\cdot$ means the multiplication in $U(\mathfrak{g}) \otimes \operatorname{End}(V)$.

Proposition 4.2. In the setting above, $\Psi$ is bijective.

Proof. By the Iwasawa decomposition we have

$$
U(\mathfrak{g}) \otimes \operatorname{End}(V) / I(\tau) \cong U(\overline{\mathfrak{n}}) \otimes U(\mathfrak{a}) \otimes \operatorname{End}(V) .
$$

Via this isomorphism the degree on $U(\mathfrak{a})$ induces a degree (denoted by $\operatorname{deg}_{\mathfrak{a}}$ ) on $U(\mathfrak{g}) \otimes \operatorname{End}(V) / I(\tau)$. Let $\mathfrak{Y} \otimes \mathcal{Z}_{1}(\mathfrak{g})$ be filtered by the total degree on $E \otimes \mathcal{Z}_{1}(\mathfrak{g})$. Notice

$$
\operatorname{deg}_{\mathfrak{a}}\left(Z \otimes 1-\left(T_{\rho-\Lambda_{\sigma}} \gamma(Z)\right)^{-1} \otimes 1+I(\tau)\right)<\operatorname{deg}(Z \otimes 1),
$$

for $Z \in \mathcal{Z}_{1}(\mathfrak{g})$, and each $\sigma \in \tau$. So $\Psi$ preserves the filtrations. It also follows that the graded map

$$
g r \Psi: U(\overline{\mathfrak{n}}) \otimes g r\left(E \otimes \mathcal{Z}_{1}(\mathfrak{g})\right) \otimes \operatorname{End}(V) \rightarrow U(\overline{\mathfrak{n}}) \otimes U(\mathfrak{a}) \otimes \operatorname{End}(V)
$$

associated to $\Psi$, is given by

$$
u \otimes e \otimes Z \otimes T \rightarrow u \cdot e \cdot\left(T_{\rho-\Lambda_{\sigma}} \gamma(Z)\right)^{-} \otimes T,
$$

for $u \in U(\overline{\mathfrak{n}}), e \in E, Z \in \mathcal{Z}_{1}(\mathfrak{g})$, and $T \in \operatorname{Hom}(V(\sigma), V)$ (here we use Proposition 1.15). This is bijective because of Proposition 4.1.

Corollary 4.3. (i) $\Psi$ maps $\mathfrak{Y} \otimes I(\lambda, \Lambda)$ onto $J(\lambda, \Lambda)$ modulo $I(\tau)$; (ii) for each $u \in U(\mathfrak{g}) \otimes \operatorname{End}(V)$ there exists a unique $y \in \mathfrak{Y}$, such that $u-y \in J(\lambda, \Lambda)$.

Proof. See Corollary 5.2 in [BS].

From the corollary we obtain a linear bijection $b_{\lambda}$ of $\mathfrak{Y}_{\lambda, \Lambda}$ onto $\mathfrak{Y}$, defined by $u-b_{\lambda}(u+J(\lambda, \Lambda)) \subset J(\lambda, \Lambda)$. Through this bijection $\mathfrak{Y}$ is equipped with a $(\mathfrak{g}, K)$ module structure from $\mathfrak{Y}_{\lambda, \Lambda}$, by making $b_{\lambda}$ a morphism of modules. Recall the $\mathfrak{g}$ action on $\mathfrak{Y}_{\lambda, \Lambda}$ is induced from left multiplication in $U(\mathfrak{g})$, and the $K$ action is induced from the following $K$ action on $U(\mathfrak{g}) \otimes \operatorname{End}(V)$,

$$
k .(u \otimes T)=\operatorname{Ad}(k) u \otimes T \tau\left(k^{-1}\right),
$$

for each $k \in K, u \in U(\mathfrak{g})$, and $T \in \operatorname{End}(V)$. Notice the difference from the action we use to define $U(\mathfrak{g})^{K}$.

Let $\tau_{\lambda}$ denote the resulting $\mathfrak{g}$ action on $\mathfrak{Y}$. Notice the action of $\overline{\mathfrak{n}}$ on $\mathfrak{Y}$ is just the left multiplication. The action of $\mathfrak{a}$ can be determined as follows: Let 
$y \in \mathfrak{Y} \subset U(\mathfrak{g}) \otimes \operatorname{End}(V), H \in \mathfrak{a}$; then $H \cdot y$ can be written (modulo $I(\tau)$ ) as $\Psi\left(\sum y_{i} \otimes Z_{i}\right)$ according to Proposition 4.2. Then by the definition of $\tau_{\lambda}$ we have

$$
\tau_{\lambda}(H) y=\sum \gamma\left(Z_{i}\right)(\lambda-\Lambda) y_{i} .
$$

For each $k \in \mathbb{N}$, let $\overline{\mathfrak{n}}^{k}$ be the linear span of $k$ times product of $\overline{\mathfrak{n}}$ in $U(\overline{\mathfrak{n}})$. Then $\tau_{\lambda}$ induces a representation $\tau_{\lambda}^{k}$ of $\mathfrak{a}+\mathfrak{m}$ on the finite dimensional space $\mathfrak{Y} / \overline{\mathfrak{n}}^{k} \mathfrak{Y}$. In particular $\tau_{\lambda}^{1}$ is a representation of $\mathfrak{a}+\mathfrak{m}$ on $\mathfrak{Y} / \overline{\mathfrak{n}} \mathfrak{Y} \cong E \otimes \operatorname{End}(V)$. By $(*)$ we know $\tau_{\lambda}$ and $\tau_{\lambda}^{k}$ are holomorphic in $\lambda$.

Let $\left\{\lambda_{1}, \ldots, \lambda_{l}\right\}$ be the set of weights of $\tau_{\lambda}^{1}$ restricted to $\mathfrak{a}$, and $\Lambda_{k} \subset-\mathbb{N} \cdot \Delta$ an enumeration of the weights of the $\mathfrak{a}$-module $U(\overline{\mathfrak{n}}) / \overline{\mathfrak{n}}^{k} U(\overline{\mathfrak{n}})$.

Proposition 4.4. For each $k \in \mathbb{N}, k \geq 1$, the set of weights of $\left(\tau_{\lambda}^{k}, \mathfrak{a}\right)$ is

$$
\left\{\lambda_{i}+\mu \mid i=1, \ldots, l, \mu \in \Lambda_{k}\right\} .
$$

Proof. By induction on $k$. It is trivial for $k=1$. For $k>1$, the induction step is a consequence of the following two exact sequences of $\mathfrak{a}$-modules.

$$
\begin{aligned}
0 & \rightarrow \overline{\mathfrak{n}}^{k-1} U(\overline{\mathfrak{n}}) / \overline{\mathfrak{n}}^{k} U(\overline{\mathfrak{n}}) \otimes \mathfrak{Y}_{\lambda, \Lambda} \rightarrow \mathfrak{Y}_{\lambda, \Lambda} / \overline{\mathfrak{n}}^{k} \mathfrak{Y}_{\lambda, \Lambda} \rightarrow \mathfrak{Y}_{\lambda, \Lambda} / \overline{\mathfrak{n}}^{k-1} \mathfrak{Y}_{\lambda, \Lambda} \rightarrow 0, \\
0 & \rightarrow \overline{\mathfrak{n}}^{k-1} U(\overline{\mathfrak{n}}) / \overline{\mathfrak{n}}^{k} U(\overline{\mathfrak{n}}) \rightarrow U(\overline{\mathfrak{n}}) / \overline{\mathfrak{n}}^{k} U(\overline{\mathfrak{n}}) \rightarrow U(\overline{\mathfrak{n}}) / \overline{\mathfrak{n}}^{k-1} U(\overline{\mathfrak{n}}) \rightarrow 0 .
\end{aligned}
$$

Let $\bar{V}_{k}=\mathfrak{Y} / \overline{\mathfrak{n}}^{k} \mathfrak{Y}$, and $\widetilde{V}_{k}$ be a finite dimensional subspace of $\mathfrak{Y}$ mapped bijectively onto $\bar{V}_{k}$ by the canonical projection. Let $\pi: \widetilde{V}_{k} \rightarrow \bar{V}_{k}$ be the restriction of the canonical projection. Define $m: \mathfrak{Y} \rightarrow U(\mathfrak{g}) \otimes \operatorname{End}(V)$ by

$$
m(u \otimes e \otimes T)=(u \cdot e) \otimes T,
$$

for $u \in U(\overline{\mathfrak{n}}), e \in E$, and $T \in \operatorname{End}(V)$.

Let $V_{k}$ be the image of $\widetilde{V}_{k}$ under $m$, and $\eta: V_{k} \rightarrow \widetilde{V}_{k}$ be the inverse of $m \mid \widetilde{V}_{k}$. Let $\mathcal{Z}(\mathfrak{a}+\mathfrak{m})$ be the center of $U(\mathfrak{a}+\mathfrak{m})$.

Proposition 4.5. For $k \in \mathbb{N}, k \geq 1$, there exist

(i) an algebra homomorphism $b_{k}(\lambda, \cdot): \mathcal{Z}(\mathfrak{a}+\mathfrak{m}) \rightarrow \operatorname{End}\left(V_{k}\right)$,

(ii) a linear map $y_{\lambda}: \mathcal{Z}(\mathfrak{a}+\mathfrak{m}) \otimes V_{k} \rightarrow \overline{\mathfrak{n}}^{k} U(\mathfrak{a}+\overline{\mathfrak{n}}) \otimes \operatorname{End}(V)$, both depending polynomially on $\lambda$, such that for each $\lambda \in \mathfrak{a}_{\mathbb{C}}^{*}, D \in \mathcal{Z}(\mathfrak{a}+\mathfrak{m})$, and $v \in V_{k}$,

$$
D v-b_{k}(\lambda, D) v-y_{\lambda}(D, v) \in J(\lambda, \Lambda) .
$$

Proof. Let $p_{\lambda}: U(\mathfrak{g}) \otimes \operatorname{End}(V) \rightarrow \mathfrak{Y}$ be the map defined by

$$
p_{\lambda}(u \otimes T)=\tau_{\lambda}(u)(1 \otimes 1 \otimes T),
$$

for $u \in U(\mathfrak{g})$, and $T \in \operatorname{End}(V)$. For $D \in \mathcal{Z}(\mathfrak{a}+\mathfrak{m}), \tilde{v} \in \widetilde{V}_{k}$ we define the maps

$$
\begin{aligned}
& \tilde{b}_{k}(\lambda, D)=\pi^{-1} \circ \tau_{\lambda}^{k} \circ \pi \in \text { End } \widetilde{V}_{k}, \\
& \tilde{y}_{\lambda}(D, \tilde{v})=p_{\lambda}((D \otimes 1) \cdot m(\tilde{v}))-m\left(\tilde{b}_{k}(\lambda, D) \tilde{v}\right) \in \mathfrak{Y} .
\end{aligned}
$$

Then $b_{k}(\lambda, \cdot)$ and $y_{\lambda}$ are defined by

$$
b_{k}(\lambda, D)=m \circ \tilde{b}_{k}(\lambda, D) \circ \eta, \quad y_{\lambda}(D, v)=m\left(\tilde{y}_{\lambda}(D, \eta(v))\right),
$$

for $D \in \mathcal{Z}(\mathfrak{a}+\mathfrak{m}), v \in V_{k}$.

Corollary 4.6. As a representation of $\mathfrak{a}, b_{k}(\lambda, \cdot)$ has the same weights as $\left(\tau_{\lambda}^{k}, \mathfrak{a}\right)$, i.e. $\left\{\lambda_{i}+\mu \mid i=1, \ldots, l, \mu \in \Lambda_{k}\right\}$. 
Proof. By definition $b_{k}(\lambda, D)=m \circ \tilde{b}_{k}(\lambda, D) \circ \eta$, and $\eta=\left(m \mid \widetilde{V}_{k}\right)^{-1}$. So $b_{k}(\lambda, \cdot)$ has the same weights as $\tilde{b}_{k}(\lambda, \cdot)$. Since $\tilde{b}_{k}(\lambda, \cdot)=\pi^{-1} \circ \tau_{\lambda}^{k} \circ \pi$, the proof is complete.

Let $V_{k}^{*}$ be the dual space of $V_{k}$, and $b_{k}^{*}(\lambda, \cdot)$ be the transpose of $b_{k}(\lambda, \cdot)$. For each weight $\xi$ of $b_{k}^{*}(\lambda, \cdot)$ we denote by $P_{\lambda, \xi}$ the projection map from $V_{k}^{*}$ onto the generalized weight space of $\xi$, along the remaining generalized weight spaces. We now consider the holomorphic dependence of $P_{\lambda, \xi}$ on $\lambda$.

Proposition 4.7. There exists for each $\lambda \in \mathfrak{a}_{\mathbb{C}}^{*}$, and each weight $\xi$ a unique polynomial $q_{\lambda, \xi}$ on $\mathfrak{a}$ with values in $\operatorname{End}\left(V_{k}^{*}\right)$, such that

$$
\begin{aligned}
P_{\lambda, \xi} q_{\lambda, \xi}(H) P_{\lambda, \xi} & =q_{\lambda, \xi}(H), \\
\exp b_{k}^{*}(\lambda, H) & =\sum_{\xi} e^{\xi(H)} q_{\lambda, \xi}(H),
\end{aligned}
$$

for $H \in \mathfrak{a}$.

Proof. Let $V_{k}^{*}(\xi)$ be the generalized weight space of $\xi$. Then the restriction of $b_{k}^{*}(\lambda, \cdot)$ to $V_{k}^{*}(\xi)$ gives a representation of $\mathfrak{a}$. $\mathfrak{a}$ is abelian so in particular solvable. Hence by Lie's theorem one can find a basis such that $b_{k}^{*}(\lambda, H) \mid V_{k}^{*}(\xi)$ corresponds to an upper triangular matrix, for each $H \in \mathfrak{a}$. The diagonal entries are $\xi(H)$. So there exists a unique polynomial $q_{\lambda, \xi}(H)$ on $\mathfrak{a}$ with values in $\operatorname{End}\left(V_{k}^{*}\right)$, such that

$$
\exp b_{k}^{*}(\lambda, H) \mid V_{k}^{*}(\xi)=e^{\xi(H)} q_{\lambda, \xi}(H) .
$$

Let $F$ be an $N$-dimensional complex vector space, and $\tau_{z}$ a family of representations of $\mathfrak{a}$ in $F$, depending on a parameter $z \in \mathbb{C}^{n}$. For each weight $\xi$ of $\tau_{z}$ let $P_{z, \xi}$ be the projection map from $F$ onto the generalized weight space $V(\xi)$, along the remaining generalized weight spaces. Fix $z_{0} \in \mathbb{C}^{n}$, and $\xi_{0}$ a weight of $\tau_{z_{0}}$.

Lemma 4.8. Given any neighborhood $N\left(\xi_{0}\right)$ of $\xi_{0}$ there exist a neighborhood $V\left(\xi_{0}\right)$ of $\xi_{0}$ in $N\left(\xi_{0}\right)$, and a neighborhood $\Omega\left(z_{0}\right)$ of $z_{0}$, such that

$$
P(z)=\sum_{\xi \in V\left(\xi_{0}\right)} P_{z, \xi} \in \operatorname{End}(F)
$$

is holomorphic in $z$ in $\Omega\left(z_{0}\right)$.

Proof. We use the argument in Chapter II in [Kato]. First let us consider the case where $\operatorname{dim} \mathfrak{a}=1$. Pick a nonzero element $H_{0} \in \mathfrak{a}$. Let

$$
T(z)=\tau_{z}\left(H_{0}\right) \in \operatorname{End}(F) .
$$

Then $\lambda_{0}=\xi_{0}\left(H_{0}\right)$ is an eigenvalue of $T\left(z_{0}\right)=\tau_{z_{0}}\left(H_{0}\right)$. Define

$$
R(z, \lambda)=(T(z)-\lambda)^{-1},
$$

for $z \in \mathbb{C}^{n}$, and $\lambda \in \mathbb{C}$. By Theorem 1.5 in Section 3 of Chapter II in [Kato], $R(z, \lambda)$ is holomorphic in the two variables $z$ and $\lambda$ in each domain where $\lambda$ is not an eigenvalue of $T(z)$. Moreover, for each $\left(z_{1}, \lambda\right)$ in such a domain,

$$
R(z, \lambda)=R\left(z_{1}, \lambda\right)+\sum_{I \in \mathbb{N}^{n}} R_{I}(\lambda)\left(z-z_{1}\right)^{I}
$$

where $R_{I}(\lambda)$ are determined by $R\left(z_{1}, \lambda\right)$, and they are holomorphic in $\lambda$. This is called the second Neumann series for the resolvent. It is uniformly convergent for sufficiently small $z-z_{1}$ and $\lambda \in \Gamma$ if $\Gamma$ is a compact subset of the resolvent set of $T\left(z_{1}\right)$. 
Let $\Gamma$ be a closed positively oriented curve in the resolvent set of $T\left(z_{0}\right)$ enclosing $\lambda_{0}$ but no other eigenvalues of $T\left(z_{0}\right)$. Then

$$
P(z)=-\frac{1}{2 i \pi} \int_{\Gamma} R(z, \lambda) d \lambda
$$

is holomorphic in $z$, for $z-z_{0}$ sufficiently small.

It is easy to see $P(z)$ is equal to the sum of the eigenprojections for all eigenvalues of $T(z)$ lying inside $\Gamma$. This basically takes care of the case $\operatorname{dim} \mathfrak{a}=1$. In general we choose a basis $e_{1}, \ldots, e_{m}$ for $\mathfrak{a}$. We can duplicate the above process to $T_{i}(z)=\tau_{z}\left(e_{i}\right)$, for $i=1, \ldots, m$. Thus we get $P_{i}(z), i=1, \ldots, m$. Then the composition of $P_{i}$ is our $P(z)$.

Fix $\lambda_{0} \in \mathfrak{a}_{\mathbb{C}}^{*}$, and $\xi_{0}$ a weight of $b_{k}^{*}\left(\lambda_{0}, \cdot\right)$. For each $\lambda \in \mathfrak{a}_{\mathbb{C}}^{*}$, let

$$
\Xi(\lambda)=\left\{w(\lambda-\Lambda)+\Lambda_{\sigma}-\rho-\mu \in X(\lambda, \Lambda) \mid w\left(\lambda_{0}-\Lambda\right)+\Lambda_{\sigma}-\rho-\mu=\xi_{0}\right\} .
$$

Proposition 4.9. There exist a neighborhood $\Omega_{0}\left(\lambda_{0}\right)$ of $\lambda_{0}$ and a neighborhood $V\left(\xi_{0}\right)$ of $\xi_{0}$, such that

$$
P(\lambda)=\sum_{\xi \in V\left(\xi_{0}\right)} P_{\lambda, \xi} \in \operatorname{End}\left(V_{k}^{*}\right)
$$

is holomorphic in $\Omega_{0}\left(\lambda_{0}\right)$, and

$$
\left\{\xi \in V\left(\xi_{0}\right) \mid \xi \text { is a weight of } b_{k}^{*}(\lambda, \cdot)\right\} \cap X(\lambda, \Lambda) \subset \Xi(\lambda) .
$$

Proof. It follows at once from Lemma 4.8 .

\section{EXISTENCE OF ASYMPtotic EXPANSION}

The methods we use in this section are similar to those used in [Ban], Section 12. Also see [BS], Section 6 .

Fix $\lambda \in \mathfrak{a}_{\mathbb{C}}^{*}, H_{0} \in \mathfrak{a}^{+}$and $r \in \mathbb{R}$. If $A_{1}, A_{2}$ are Banach spaces we denote by $B\left(A_{1}, A_{2}\right)$ the Banach space of bounded linear operators from $A_{1}$ to $A_{2}$.

Proposition 5.1. There exist, for each $N \in \mathbb{R}$,

(a) open neighborhoods $\Omega$ of $\lambda_{0} \in \mathfrak{a}_{\mathbb{C}}^{*}$ and $U$ of $H_{0} \in \mathfrak{a}^{+}$,

(b) constants $k, q \in \mathbb{N}, r^{\prime} \geq r$, and $C, \varepsilon>0$,

(c) a continuous map

$$
\Phi: \Omega \times U \rightarrow B\left(C_{r}^{q}(G, V), V_{k}^{*} \otimes C_{r^{\prime}}(G, V)\right),
$$

holomorphic in the first variable, and

(d) a linear form $\eta \in\left(V_{k}^{*}\right)^{*}$, such that

(i) $\Phi(\lambda, H)$ intertwines the left actions of $G$ on $C_{r}^{q}(G, V)$ and $C_{r^{\prime}}(G, V)$, for all $(\lambda, H) \in \Omega \times U$, and (ii)

$$
\left\|R_{\exp t H} f-\left(\eta \circ \exp b_{k}^{*}(\lambda, t H) \otimes 1\right) \Phi(\lambda, H) f\right\|_{r^{\prime}} \leq C\|f\|_{q, r} e^{(N-\varepsilon) t},
$$

for $f \in \mathcal{E}_{\lambda-\Lambda} \operatorname{Ind}_{K}^{G}(\tau) \cap C_{r}^{q}(G, V), \lambda \in \Omega, H \in U, t \geq 0$.

Proof. In the same way as for Proposition 12.6 in [Ban].

We now begin the proof of Theorem 3.2. Using Proposition 4.7 we can write

$$
\left(\eta \circ \exp b_{k}^{*}(\lambda, t H) \otimes 1\right) \Phi(\lambda, H)=\sum_{\xi} p_{\lambda, \xi}(H, t) e^{t \xi(H)},
$$


for $\lambda \in \Omega, H \in U, t \geq 0$, where the summation extends to the weights $\xi$ of $b_{k}^{*}(\lambda, \cdot)$ which by Corollary 4.6 is the set

$$
\left\{\lambda_{i}+\mu \mid i=1, \ldots, l, \mu \in \Lambda_{k}\right\},
$$

and where $p_{\lambda, \xi}(H, t)=\left(\eta \circ q_{\lambda, \xi}(t H) \otimes 1\right) \Phi(\lambda, H) \in B\left(C_{r}^{q}, C_{r^{\prime}}\right)$, which is continuous in $H$ and polynomial in $t$. From (d) (ii) of Proposition 5.1 we have

$$
\left\|R_{\exp t H} f-\sum_{\xi} e^{t \xi(H)} p_{\lambda, \xi}(H, t) f\right\|_{r^{\prime}} \leq C\|f\|_{q, r} e^{t(N-\varepsilon)},
$$

for $f \in \mathcal{E}_{\lambda-\Lambda} \operatorname{Ind}_{K}^{G}(\tau) \cap C_{r}^{q}(G, V)$. Since $N$ is arbitrary we have for each $g \in G$,

$$
f(g \exp t H) \sim \sum_{\xi \in \widetilde{X}(\lambda, \Lambda)}\left(p_{\lambda, \xi}(H, t) f\right)(g) e^{t \xi(H)} \quad(t \rightarrow \infty) .
$$

Here $\widetilde{X}(\lambda, \Lambda)=\left\{\lambda_{i}+\mu \mid i=1, \ldots, l, \mu \in-\mathbb{N} \cdot \Delta\right\}$.

Lemma 5.2. Let $X \subset \mathfrak{a}_{\mathbb{C}}^{*}$ and $f: \mathfrak{a}^{+} \rightarrow V$. Assume that for each $H_{0} \in \mathfrak{a}^{+}$there is a given formal expansion

$$
\sum_{\xi \in X} p_{\xi, H_{0}}(H, t) e^{t \xi(H)}
$$

which is an asymptotic expansion for $f$ at $H_{0}$. Then for each $\xi \in X$ there exists a unique continuous function $p_{\xi}: \mathfrak{a}^{+} \rightarrow V$ such that for each $H_{0} \in \mathfrak{a}^{+}$there is a neighborhood $U$ with

$$
p_{\xi, H_{0}}(H, t)=p_{\xi}(t H)
$$

for $H \in U$, and $t>0$.

Proof. See Corollary 3.4 in [BS].

As can be seen in the proof of Proposition 12.6 in [Ban], for $t>0, H \in U$ with $t H \in U, \Phi(\lambda, t H)=\Phi(\lambda, H)$. Thus for $t>0, H \in U$ with $t H \in U$, $\left(p_{\lambda, \xi}(H, t) f\right)(g)=\left(p_{\lambda, \xi}(t H, 1) f\right)(g)$. By Lemma 5.2, for $\lambda \in \mathfrak{a}_{\mathbb{C}}^{*}, r \in \mathbb{R}$, and $\xi \in \widetilde{X}(\lambda, \Lambda)$, there exist constants $r^{\prime} \in \mathbb{R}, q \in \mathbb{N}$, and a unique continuous map $p_{\lambda, \xi}(\cdot, \cdot, \cdot): \mathfrak{a}^{+} \rightarrow B\left(\mathcal{E}_{\lambda-\Lambda} \operatorname{Ind}_{K}^{G}(\tau) \cap C_{r}^{q}(G, V), C_{r^{\prime}}(G, V)\right)$, such that

$$
f(g \exp t H) \sim \sum_{\xi \in \widetilde{X}(\lambda, \Lambda)} p_{\lambda, \xi}(f, g, t H) e^{t \xi(H)} \quad(t \rightarrow \infty),
$$

at every $H_{0} \in \mathfrak{a}$, for $f \in \mathcal{E}_{\lambda-\Lambda} \operatorname{Ind}_{K}^{G}(\tau) \cap C_{r}^{q}(G, V)$.

To complete the proof of Theorem 3.2 it remains to show (1) we can replace $\widetilde{X}(\lambda, \Lambda)$ by $X(\lambda, \Lambda),(2) p_{\lambda, \xi}(f, g, H)$ is a polynomial in $H$ with order $\leq d$. We shall finish the proof in the next section. We now consider the holomorphic dependence in $\lambda$ in order to prove Theorem 3.3.

Let $r \in \mathbb{R}$ and $\Omega$ be an open set in $\mathfrak{a}_{\mathbb{C}}^{*}$. Let $\left\{f_{\lambda}\right\}_{\lambda \in \Omega}$ be a holomorphic family in $C_{r}^{\infty}(G, V)$, and $f_{\lambda} \in \mathcal{E}_{\lambda-\Lambda}^{\infty} \operatorname{Ind}_{K}^{G}(\tau)$, for each $\lambda \in \Omega$. We now study the asymptotic expansion of $f_{\lambda}$. Fix $\lambda_{0} \in \Omega$, and $\xi_{0} \in \tilde{X}\left(\lambda_{0}, \Lambda\right)$.

Proposition 5.3. There exist a neighborhood $\Omega\left(\lambda_{0}\right)$ of $\lambda_{0}$ in $\Omega$ and a neighborhood $V\left(\xi_{0}\right)$ of $\xi_{0}$ in $\mathfrak{a}_{\mathbb{C}}^{*}$, such that

$$
(\lambda, H) \rightarrow \sum_{\xi \in V\left(\xi_{0}\right)} p_{\lambda, \xi}\left(f_{\lambda}, \cdot, H\right) e^{\xi(H)}
$$


is continuous from $\Omega\left(\lambda_{0}\right) \times U$ to $C_{r^{\prime}}^{q^{\prime}}(G, V)$ for some $q^{\prime} \in \mathbb{N}, r^{\prime} \in \mathbb{R}$, and in addition holomorphic in $\lambda$. Moreover, we can choose $V\left(\xi_{0}\right)$ such that $V\left(\xi_{0}\right) \cap X(\lambda, \Lambda) \subset \Xi(\lambda)$.

Proof. It follows from Proposition 4.9.

\section{Differential equations for the COefFicients}

In this section we derive certain differential equations for the vector-valued functions $p_{\lambda, \xi}(f, g, \cdot)$ on $\mathfrak{a}^{+}$, where $f \in \mathcal{E}_{\lambda-\Lambda}^{\infty} \operatorname{Ind}_{K}^{G}(\tau)$, and $g \in G$.

Fix $Z \in \mathcal{Z}(\mathfrak{g})$, and $D=\mu(Z) \in Z_{\tau}$. We can choose finitely many $x_{i}$ in $\overline{\mathfrak{n}} U(\overline{\mathfrak{n}})$, and $v_{i} \in U(\mathfrak{a}) \otimes \operatorname{End}(V)$, such that

$$
Z-\widetilde{\Gamma}_{1}(Z \otimes 1)-\sum x_{i} v_{i} \in I(\tau)
$$

and $\operatorname{ad}(\mathfrak{a})$ acts on $x_{i}$ by a weight $-\eta_{i} \neq 0$, where $\eta_{i} \in \mathbb{N} \cdot \Delta$, and $v_{i}, \widetilde{\Gamma}_{1}(Z \otimes 1) \in$ $U(\mathfrak{a}) \otimes \operatorname{End}(V)$ which can be interpreted as differential operators with constant coefficients on $C^{\infty}(\mathfrak{a}, V)$.

Proposition 6.1. Let $f \in \mathcal{E}_{\lambda-\Lambda}^{\infty} \operatorname{Ind}_{K}^{G}(\tau)$. Then the functions $p_{\lambda, \xi}(f, \cdot, \cdot) e^{\xi}$ from $G \times \mathfrak{a}^{+}$to $V$ satisfy the following recursive equations:

$$
\begin{aligned}
& 1 \otimes \partial\left(\widetilde{\Gamma}_{1}(Z \otimes 1)-\gamma(Z)(\lambda-\Lambda)\right)\left(p_{\lambda, \xi}(f, \cdot, \cdot) e^{\xi}\right) \\
& \quad=-\sum_{i, \xi+\eta_{i} \in \widetilde{X}(\lambda, \Lambda)} R_{x^{i}} \otimes e^{-\eta_{i}} \partial\left(v^{i}\right)\left(p_{\lambda, \xi+\eta_{i}}(f, \cdot, \cdot) e^{\xi+\eta_{i}}\right),
\end{aligned}
$$

for all $\xi \in \tilde{X}(\lambda, \Lambda)$.

The proof is the same as for Proposition 7.1 in [BS].

Proof of Theorem 3.2. Let

$$
V=\bigoplus_{\Lambda_{1} \in \mathfrak{t}^{*}} V\left(\Lambda_{1}\right)
$$

where $V\left(\Lambda_{1}\right)=\bigoplus_{\sigma \in \tau, \Lambda_{\sigma}=\Lambda_{1}} V(\sigma)$. Let $P\left(\Lambda_{1}\right)$ be the projection from $V$ to $V\left(\Lambda_{1}\right)$. By Corollary $1.15 \Gamma_{1}(Z \otimes 1) \mid V\left(\Lambda_{1}\right)=\left(T_{\rho-\Lambda_{1}} \gamma(Z)\right)^{-} \otimes \mathrm{I}_{V\left(\Lambda_{1}\right)}$. For $\xi_{1}, \xi_{2} \in \mathfrak{a}_{\mathbb{C}}^{*}$, we say $\xi_{1} \prec \xi_{2}$ if there exists $\eta \in \mathbb{N} \cdot \Delta$ such that $\xi_{2}=\xi_{1}+\eta$. This defines a partial order on $\mathfrak{a}_{\mathbb{C}}^{*}$. For each $f \in \mathcal{E}_{\lambda-\Lambda}^{\infty} \operatorname{Ind}_{K}^{G}(\tau)$, define $E(\lambda, \Lambda, f)$ by

$$
E(\lambda, \Lambda, f)=\left\{\xi \in \widetilde{X}(\lambda, \Lambda) \mid p_{\lambda, \xi}(f, \cdot, \cdot) \not \equiv 0\right\} .
$$

Let $E_{L}(\lambda, \Lambda, f)$ denote the set of maximal elements in $E(\lambda, \Lambda, f)$. Suppose $\xi \in$ $E_{L}(\lambda, \Lambda, f)$. Then $p_{\lambda, \xi}(f, \cdot, \cdot) \not \equiv 0$. So one can find $g \in G, \Lambda_{1} \in \mathfrak{t}^{*}$, such that $P\left(\Lambda_{1}\right) p_{\lambda, \xi}(f, g, \cdot) \not \equiv 0$.

Since the right-hand side of the equation in Proposition 6.1 is zero because $\xi$ is maximal in $E(\lambda, \Lambda, f)$,

$$
\partial\left(\widetilde{\Gamma}_{1}(Z \otimes 1)-\gamma(Z)(\lambda-\Lambda)\right)\left(p_{\lambda, \xi}(f, g, \cdot) e^{\xi}\right)=0 .
$$

So

$$
\partial\left(\left(T_{-\Lambda_{1}+\rho} \gamma(Z)\right)^{-}-\gamma(Z)(\lambda-\Lambda)\right)\left(P\left(\Lambda_{1}\right) p_{\lambda, \xi}(f, g, \cdot) e^{\xi}\right)=0 .
$$

We extend $p_{\lambda, \xi}(f, g, \cdot) e^{\xi}$ to a function on $\mathfrak{a}^{+}+\sqrt{-1} \mathfrak{t} \subset \mathfrak{h}=\mathfrak{a}+\sqrt{-1} \mathfrak{t}$, by abuse of notation still denoted by $p_{\lambda, \xi}(f, g, \cdot) e^{\xi}$, by the requirement that it be constant in the $\mathfrak{t}$ direction. Hence

$$
\partial\left(\left(T_{-\Lambda_{1}+\rho} \gamma(Z)\right)-\gamma(Z)(\lambda-\Lambda)\right)\left(P\left(\Lambda_{1}\right) p_{\lambda, \xi}(f, g, \cdot) e^{\xi}\right)=0 .
$$


So

$$
\partial((\gamma(Z))-\gamma(Z)(\lambda-\Lambda))\left(P\left(\Lambda_{1}\right) p_{\lambda, \xi}(f, g, \cdot) e^{\xi-\Lambda_{1}+\rho}\right)=0 .
$$

By Theorem 3.13, Chapter III in [Helg1], $P\left(\Lambda_{1}\right) p_{\lambda, \xi}(f, g, \cdot) e^{\xi-\Lambda_{1}+\rho}=\sum q_{i} e^{\mu_{i}}$, where $q_{i}$ are polynomials on $\mathfrak{h}, \mu_{i} \in \mathfrak{h}_{\mathbb{C}}^{*}$. Recall that $p_{\lambda, \xi}(f, g, t H)$ is a polynomial in $t$. We conclude $P\left(\Lambda_{1}\right) p_{\lambda, \xi}(f, g, \cdot)$ is a polynomial on $\mathfrak{h}$, and

$$
\xi-\Lambda_{1}+\rho=w(\lambda-\Lambda),
$$

for some $w \in \widetilde{W}$. Also $P\left(\Lambda_{1}\right) p_{\lambda, \xi}(f, g, \cdot)$ is a $\widetilde{W}(w(\lambda-\Lambda))$-harmonic, where $\widetilde{W}(\mu)=$ $\{w \in \widetilde{W} \mid w \mu=\mu\}$, for each $\mu \in \mathfrak{h}_{\mathbb{C}}^{*}$. So

$$
\operatorname{deg}\left(P\left(\Lambda_{1}\right) p_{\lambda, \xi}(f, g, \cdot)\right) \leq d
$$

Here $d$ is the number of elements in $\Sigma^{+}\left(\mathfrak{g}_{\mathbb{C}}, \mathfrak{h}_{\mathbb{C}}\right)$. It follows that we can replace $\widetilde{X}(\lambda, \Lambda)$ by $X(\lambda, \Lambda)$ since $E_{L}(\lambda, \Lambda, f) \subset X(\lambda, \Lambda)$.

By induction on $\xi$ using Proposition 6.1 one can easily show $p_{\lambda, \xi}(f, g, \cdot)$ is a polynomial with degree $\leq d$. Note we only need to show it for $g=e$. So this completes the proof of Theorem 3.2.

The proof of Theorem 3.3 follows from Proposition 5.3.

\section{LEADING EXPONENTS}

We further consider the properties of a leading term in the asymptotic expansion of $f \in \mathcal{E}_{\lambda-\Lambda}^{\infty} \operatorname{Ind}_{K}^{G}(\tau)$.

Proposition 7.1. For each $\xi \in E_{L}(\lambda, \Lambda, f)$, man $\in B, H \in \mathfrak{a}$, and $g \in G$,

$$
p_{\lambda, \xi}(f, g m a n, H)=e^{\xi(\log a)} \tau(m)^{-1} p_{\lambda, \xi}(f, g, H+\log a) .
$$

Proof. The same as for Theorem 8.4 in [BS].

Let $\alpha^{\vee}=2 \alpha /\langle\alpha, \alpha\rangle$. We introduce conditions on $\lambda-\Lambda$ and $\lambda$ as follows:

$$
\begin{gathered}
\mathfrak{A}_{1}=\left\{\lambda-\Lambda\left|\lambda \in \mathfrak{a}_{\mathbb{C}}^{*}, \Lambda \in \mathfrak{t}_{\mathbb{C}}^{*},\left\langle\lambda-\Lambda, \alpha^{\vee}\right\rangle \notin \mathbb{Z}, \forall \alpha \in \Sigma\left(\mathfrak{g}_{\mathbb{C}}, \mathfrak{h}_{\mathbb{C}}\right), \alpha\right| \mathfrak{a} \neq 0\right\}, \\
\mathfrak{A}_{2}=\left\{\lambda \in \mathfrak{a}_{\mathbb{C}}^{*} \mid\left\langle\lambda, \beta^{\vee}\right\rangle \notin-\mathbb{N}, \forall \beta \in \Sigma^{+}(\mathfrak{g}, \mathfrak{a})\right\} .
\end{gathered}
$$

Let

$$
\widetilde{W}_{0}=\{w \in \widetilde{W}|w| \mathfrak{a}=\mathrm{id}\}, \quad \widetilde{W}_{1}=\{w \in \widetilde{W} \mid w \mathfrak{a}=\mathfrak{a}\} .
$$

Proposition 7.2. Suppose $\lambda-\Lambda \in \mathfrak{A}_{1}$. We have

(i) if $w(\lambda-\Lambda)=\lambda-\Lambda$ for some $w \in \widetilde{W}$, then $w \in \widetilde{W}_{0}$;

(ii) if there exist $w \in \widetilde{W}, \sigma \in \tau$ such that

$$
\left(w(\lambda-\Lambda)+\Lambda_{\sigma}\right) \mid \mathfrak{t}=0,
$$

then $w \in \widetilde{W}_{1}$, and $\Lambda_{\sigma}=w \Lambda$.

Proof. (i) Since $w(\lambda-\Lambda)=\lambda-\Lambda, w=w_{\alpha_{1}} \cdots w_{\alpha_{s}}$, where $\alpha_{j} \in \Sigma\left(\mathfrak{g}_{\mathbb{C}}, \mathfrak{h}_{\mathbb{C}}\right)$, and $\left\langle\lambda-\Lambda, \alpha_{j}\right\rangle=0$. Then we conclude $\alpha_{j} \mid \mathfrak{a}=0$ from $\mathfrak{A}_{1}$. So $w \in \widetilde{W}_{0}$. (ii) For any $\beta \in$ $\Sigma\left(\mathfrak{g}_{\mathbb{C}}, \mathfrak{h}_{\mathbb{C}}\right)$ with $\beta \mid \mathfrak{a}=0$, we have $\left\langle w(\lambda-\Lambda)+\Lambda_{\sigma}, \beta\right\rangle=0$ since $\left(w(\lambda-\Lambda)+\Lambda_{\sigma}\right) \mid \mathfrak{t}=0$. Hence

$$
\begin{aligned}
& \frac{2\left\langle\lambda-\Lambda, w^{-1} \beta\right\rangle}{\langle\beta, \beta\rangle}=-\frac{2\left\langle\Lambda_{\sigma}, \beta\right\rangle}{\langle\beta, \beta\rangle}, \\
& \frac{2\left\langle\lambda-\Lambda, w^{-1} \beta\right\rangle}{\left\langle w^{-1} \beta, w^{-1} \beta\right\rangle}=-\frac{2\left\langle\Lambda_{\sigma}, \beta\right\rangle}{\langle\beta, \beta\rangle} .
\end{aligned}
$$


The right-hand side being integral forces $w^{-1} \beta \mid \mathfrak{a}=0$. This shows $w$ preserves $\mathfrak{t}$. Therefore $w$ preserves $\mathfrak{a}$. Trivially $\Lambda_{\sigma}=w \Lambda$.

Proposition 7.3. Let $f \in \mathcal{E}_{\lambda-\Lambda}^{\infty} \operatorname{Ind}_{K}^{G}(\tau)$. Suppose $\lambda-\Lambda \in \mathfrak{A}_{1}$, and $\xi$ in $E_{L}(\lambda, \Lambda, f)$. Then $\xi \in W \lambda-\rho$, and $p_{\lambda, \xi}(f, g, \cdot)$ is constant in $\mathfrak{a}$ for each $g \in G$.

Proof. In the last section we showed if $P\left(\Lambda_{\sigma}\right) p_{\lambda, \xi}(f, g, \cdot) \not \equiv 0$, then there exists $w \in \widetilde{W}$, such that $\xi-\Lambda_{\sigma}+\rho=w(\lambda-\Lambda)$. So

$$
\left(w(\lambda-\Lambda)+\Lambda_{\sigma}\right) \mid \mathfrak{t}=0 .
$$

By Proposition 7.2 (ii) $w \in \widetilde{W}_{1}$. So $\xi+\rho=w \lambda$. Hence $\xi \in W \lambda-\rho$.

We also showed that $P\left(\Lambda_{\sigma}\right) p_{\lambda, \xi}(f, g, \cdot)$ is $\widetilde{W}(w(\lambda-\Lambda))$-harmonic. Since $w \in$ $\widetilde{W}_{1}, w(\lambda-\Lambda) \in \mathfrak{A}_{1}$. By Proposition 7.2 (i) $\widetilde{W}(w(\lambda-\Lambda)) \subset \widetilde{W}_{0}$. We conclude $P\left(\Lambda_{\sigma}\right) p_{\lambda, \xi}(f, g, \cdot)$ is constant in $\mathfrak{a}$. This shows $p_{\lambda, \xi}(f, g, \cdot)$ is constant in a since $\sigma \in \tau$ is arbitrary. In this case we denote it by $p_{\lambda, \xi}(f, g)$.

Corollary 7.4. If $\lambda-\rho \in E_{L}(\lambda, \Lambda, f)$, and in addition $\lambda$ is regular, i.e., $W(\lambda)=$ $\{w \in W \mid w \lambda=\lambda\}=e$, then

$$
p_{\lambda, \lambda-\rho}(f, g)=P(\Lambda) p_{\lambda, \lambda-\rho}(f, g) .
$$

Proof. If for some $\sigma \in \tau$, such that $P\left(\Lambda_{\sigma}\right) p_{\lambda, \xi}(f, g) \not \equiv 0$, then there exists $w \in \widetilde{W}_{1}$, with

$$
w \lambda=(\lambda-\rho)+\rho, w \Lambda_{\sigma}=\Lambda .
$$

$\lambda$ being regular implies $w \in \widetilde{W}_{0}$. But then $P(\Lambda)=P\left(\Lambda_{\sigma}\right)$ by definition.

By Appendix II in [KKMOOT] if $\lambda \in \mathfrak{A}_{2}$, then $\lambda-\rho$ is always maximal in $W \lambda-\rho$. So we have the following definition.

Definition 7.5. Let $\lambda-\Lambda \in \mathfrak{A}_{1}$, and $\lambda \in \mathfrak{A}_{2}$. For $f \in \mathcal{E}_{\lambda-\Lambda}^{\infty} \operatorname{Ind}_{K}^{G}(\tau), \beta_{\lambda}(f)$ is defined by

$$
\beta_{\lambda}(f)=p_{\lambda, \lambda-\rho}(f, \cdot) .
$$

We call $\beta_{\lambda}$ the boundary value map.

Theorem 7.6. Let $\lambda-\Lambda \in \mathfrak{A}_{1}, \lambda \in \mathfrak{A}_{2}$. Then

(i) $\beta_{\lambda}$ maps $\mathcal{E}_{\lambda-\Lambda, r}^{\infty} \operatorname{Ind}_{K}^{G}(\tau)$ linearly, continuously, and G-equivariantly into $C^{\infty} \operatorname{Ind}_{B}^{G}(\tau(\Lambda) \otimes(-\lambda) \otimes 1)$ for each $r \in \mathbb{R}$, where $\tau(\Lambda)$ is the restriction of $\tau$ to $M$ with representation space $V(\Lambda)$.

(ii) Let $\Omega \subset \mathfrak{a}_{\mathbb{C}}^{*}$ be open, $\left\{f_{\lambda}\right\}_{\lambda \in \Omega}$ a holomorphic family in $\mathcal{E}_{\lambda-\Lambda}^{\infty} \operatorname{Ind}_{K}^{G}(\tau)$; then $\lambda \rightarrow \beta_{\lambda}\left(f_{\lambda}\right)$ is holomorphic in $\Omega \cap \mathfrak{A}_{2}$.

Proof. (i) comes from Theorem 3.2; (ii) is a result of Theorem 3.3.

Finally we notice for certain $\lambda$ we can obtain the boundary value map by a simple limit procedure.

Lemma 7.7. Let $\lambda-\Lambda \in \mathfrak{A}_{1}$. If $\operatorname{Re}\langle\lambda, \alpha\rangle>0$, for each $\alpha \in \Sigma^{+}(\mathfrak{g}, \mathfrak{a})$, then

$$
\beta_{\lambda} f(g)=\lim _{t \rightarrow \infty} e^{(-\lambda+\rho)(t H)} f(g \exp t H),
$$

for $f \in \mathcal{E}_{\lambda-\Lambda}^{\infty} \operatorname{Ind}_{K}^{G}(\tau)$, and $H \in \mathfrak{a}^{+}$. 
Proof. The condition on $\lambda$ implies that $\operatorname{Re} \xi(H)<\operatorname{Re}(\lambda-\rho)(H)$ for all $\xi \in X(\lambda, \Lambda)$ with $\xi \neq \lambda-\rho$. Then the result follows from Theorem 3.2 and the very definition of asymptotic expansion.

For each $\phi \in C^{\infty} \operatorname{Ind}_{B}^{G}(\tau(\Lambda) \otimes(-\lambda) \otimes 1)$, we define $P_{\lambda} \phi$ by

$$
P_{\lambda} \phi(g)=\int_{K} \tau(k) \phi(g k) d k .
$$

From the proof of Theorem 1.6 we conclude $P_{\lambda} \phi \in \mathcal{E}_{\lambda-\Lambda, r} \operatorname{Ind}_{K}^{G}(\tau)$. By Example 2.2 $P_{\lambda} \phi \in \mathcal{E}_{\lambda-\Lambda, r}^{\infty} \operatorname{Ind}_{K}^{G}(\tau)$.

Corollary 7.8. Under the same conditions as in Lemma 7.7,

$$
\beta_{\lambda} P_{\lambda} \phi=C(\lambda) \phi,
$$

for each $\phi \in C^{\infty} \operatorname{Ind}_{B}^{G}(\tau(\Lambda) \otimes(-\lambda) \otimes 1)$.

Proof. By Proposition 2.4 and Lemma 7.7.

\section{The inversion of the Poisson transform}

Let $C(\lambda)$ be the generalized Harish-Chandra $C$-function given by

$$
C(\lambda)=\int_{\bar{N}} e^{-(\lambda+\rho) \mathrm{H}(\bar{n})} \tau(\mathrm{k}(\bar{n})) d \bar{n} .
$$

Recall $P_{\lambda}: C^{\infty} \operatorname{Ind}_{B}^{G}(\tau(\Lambda) \otimes(-\lambda) \otimes 1) \rightarrow \mathcal{E}_{\lambda-\Lambda}^{\infty} \operatorname{Ind}_{K}^{G}(\tau)$ is defined by

$$
P_{\lambda} \phi(g)=\int_{K} \tau(k) \phi(g k) d k .
$$

Theorem 8.1. Let $\lambda-\Lambda \in \mathfrak{A}_{1}, \lambda \in \mathfrak{A}_{2}$, and $C_{0}(\lambda)$ the restriction of $C(\lambda)$ to $V(\Lambda)$. Then

$$
\beta_{\lambda} P_{\lambda} \phi=C_{0}(\lambda) \phi
$$

for each $\phi \in C^{\infty} \operatorname{Ind}_{B}^{G}(\tau(\Lambda) \otimes(-\lambda) \otimes 1)$.

Proof. If $\operatorname{Re}\langle\lambda, \alpha\rangle>0$, for all $\alpha \in \Sigma(\mathfrak{g}, \mathfrak{a})$, then by Corollary 7.8 ,

$$
\beta_{\lambda} P_{\lambda} \phi=C_{0}(\lambda) \phi \text {. }
$$

Since $P_{\lambda} \phi$ is a holomorphic family in $\mathcal{E}_{\lambda-\Lambda}^{\infty} \operatorname{Ind}_{K}^{G}(\tau)$, by Theorem 7.6 the left-hand side is holomorphic. The right-hand side is meromorphic on $\mathfrak{a}_{\mathbb{C}}^{*}$. Hence two sides must coincide.

Corollary 8.2. If in addition we assume $\operatorname{det} C_{0}(\lambda) \neq 0$, then $\beta_{\lambda}$ is surjective. Hence $P_{\lambda}$ is injective.

Theorem 8.3. Let $\lambda-\Lambda \in \mathfrak{A}_{1}$, and $\lambda \in \mathfrak{A}_{2}$, and $\operatorname{det} C_{0}(\lambda) \neq 0$. Then $P_{\lambda}$ is bijective, and the inverse of $P_{\lambda}$ is given by $C_{0}(\lambda)^{-1} \beta_{\lambda}$.

For the proof we recall a definition which can be found in [Wall], Section 11.6. Let $\mathfrak{V}$ be a finitely generated $(\mathfrak{g}, K)$-module.

Definition 8.4. $\mathfrak{V}_{\text {mod }}^{*}$ denotes the set of all $\mu \in \mathfrak{V}^{*}$, such that there exists $d_{\mu} \in \mathbb{R}$ and for each $\nu \in \mathfrak{V}$ there exist an analytic function $f_{\mu, \nu}$ and a constant $C_{\mu, \nu}>0$ with the following properties:

(i) $L_{u} f_{\mu, \nu}(k)=\mu\left(k^{-1} \cdot(u . \nu)\right)$, for $u \in U(\mathfrak{g}), k \in K$,

(ii) $\left|f_{\mu, \nu}(g)\right| \leq C_{\mu, \nu}\|g\|^{d_{\mu}}$, for each $g \in G$. 
Recall that $\left(C^{\infty} \operatorname{Ind}_{B}^{G}(\sigma \otimes(-\lambda) \otimes 1)\right)^{\prime}$ is the strong topological dual of $C^{\infty} \operatorname{Ind}_{B}^{G}(\sigma \otimes(-\lambda) \otimes 1)$. The following result can also be found in [Wall], Section 11.7 .

Proposition 8.5. Let $\left(C^{\infty} \operatorname{Ind}_{B}^{G}(\sigma \otimes(-\lambda) \otimes 1)\right)_{K \text {-finite }}$ denote the space of $K$-finite elements in $C^{\infty} \operatorname{Ind}_{B}^{G}(\sigma \otimes(-\lambda) \otimes 1)$. Then

$$
\left(C^{\infty} \operatorname{Ind}_{B}^{G}(\sigma \otimes(-\lambda) \otimes 1)_{K \text {-finite }}\right)_{\text {mod }}^{*}=\left(C^{\infty} \operatorname{Ind}_{B}^{G}(\sigma \otimes(-\lambda) \otimes 1)\right)^{\prime} .
$$

Before we go ahead with the proof of Theorem 8.3, we mention the following result about the irreducibility of the principal series representations. Let $\sigma \in \widehat{M}$.

Lemma 8.6. As a $(\mathfrak{g}, K)$-module $C^{\infty} \operatorname{Ind}_{B}^{G}(\sigma \otimes(-\lambda) \otimes 1)_{K \text {-finite }}$ is irreducible if $\lambda-\Lambda \in \mathfrak{A}_{1}$.

Proof. This is a direct consequence of Theorem 1.1 in [SV].

Proof of Theorem 8.3. It suffices to show $\beta_{\lambda}$ is injective. Assume the opposite. Then there exists $f_{0} \in \mathcal{E}_{\lambda-\Lambda}^{\infty} \operatorname{Ind}_{K}^{G}(\tau)$, such that $\beta_{\lambda} f_{0}=0$, and $f_{0} \not \equiv 0$. We can assume $f_{0}(e) \neq 0$ since $\beta$ is $G$-equivariant. Define $f_{K}$ by

$$
f_{K}(g)=\int_{K} \operatorname{tr} \tau(k) f_{0}(k g) d k .
$$

Then $f_{K}$ is $K$-finite, and $f_{K}(e)=\frac{1}{\operatorname{dim}(\tau)} f_{0}(e) \neq 0$. Let

$$
\mathfrak{W}=L_{U(\mathfrak{g})} L_{K} f_{K} .
$$

Then $\mathfrak{W}$ is a finitely generated $(\mathfrak{g}, K)$-module. Let $\mathfrak{W}_{1}$ be an irreducible submodule of $\mathfrak{W}$. By the subrepresentation theorem and Lemma 8.4 there exists $\sigma \in \widehat{M}$, such that $\mathfrak{W}_{1} \cong C^{\infty} \operatorname{Ind}_{B}^{G}(\sigma \otimes(-\lambda) \otimes 1)_{K \text {-finite. So there is a }(\mathfrak{g}, K) \text { map }}$

$$
P_{\sigma}: C^{\infty} \operatorname{Ind}_{B}^{G}(\sigma \otimes(-\lambda) \otimes 1)_{K \text {-finite }} \rightarrow \mathfrak{W} \text {. }
$$

It is easy to see $\Lambda=\Lambda_{\sigma}$. Define $\mu \in \mathfrak{W}^{*} \otimes V$ by

$$
\mu(\nu)=\nu(e)
$$

for each $\nu \in \mathfrak{W}$.

Taking $f_{\mu, \nu}=\nu \in \mathcal{E}_{\lambda-\Lambda}^{\infty} \operatorname{Ind}_{K}^{G}(\tau)$ in Definition 8.4, we can verify that (i) and (ii) are satisfied. So $\mu \in \mathfrak{W}_{\text {mod }}^{*} \otimes V$. Hence

$$
\mu^{\sharp}=\mu \circ P_{\sigma} \in\left(C^{\infty} \operatorname{Ind}_{B}^{G}(\sigma \otimes(-\lambda) \otimes 1)_{K \text {-finite }}\right)_{\text {mod }}^{*} \otimes V .
$$

Then by Proposition 8.5,

$$
\mu^{\sharp} \in\left(C^{\infty} \operatorname{Ind}_{B}^{G}(\sigma \otimes(-\lambda) \otimes 1)\right)^{\prime} \otimes V .
$$

Now define $P_{\sigma}^{\sharp}: C^{\infty} \operatorname{Ind}_{B}^{G}(\sigma \otimes(-\lambda) \otimes 1) \rightarrow C^{\infty} \operatorname{Ind}_{K}^{G}(\tau)$ by

$$
P_{\sigma}^{\sharp} \phi(g)=\mu^{\sharp}\left(L_{g^{-1}} \phi\right) \text {. }
$$

Since $P_{\sigma}$ is a $\mathfrak{g}$ map and eigensections are analytic we can show that for $\phi$ in $C^{\infty} \operatorname{Ind}_{B}^{G}(\sigma \otimes(-\lambda) \otimes 1)_{K \text {-finite }}$

$$
P_{\sigma} \phi=P_{\sigma}^{\sharp} \phi,
$$

by showing they are identical at $e$ along with their derivatives. 
We observe that $P_{\sigma}^{\sharp}$ is a linear, continuous, and $G$-equivariant map from $C^{\infty} \operatorname{Ind}_{B}^{G}(\sigma \otimes(-\lambda) \otimes 1)$ to $C^{\infty} \operatorname{Ind}_{K}^{G}(\tau)$. By Proposition 1.8 we conclude $\sigma \in \tau$, and there exists $T \in \operatorname{Hom}_{M}\left(V_{\sigma}, V\right)$ such that $P_{\sigma}^{\sharp}=P_{T}$. Hence

$$
P_{\sigma}=P_{T}: C^{\infty} \operatorname{Ind}_{B}^{G}(\sigma \otimes(-\lambda) \otimes 1)_{K \text {-finite }} \rightarrow \mathfrak{W} \text {. }
$$

Taking $\sigma \in C^{\infty} \operatorname{Ind}_{B}^{G}(\sigma \otimes(-\lambda) \otimes 1)_{K \text {-finite }}$ such that $0 \neq f=P_{T} \phi$, then $f=P_{\lambda}(T \phi)$. Notice $T \phi \in C^{\infty} \operatorname{Ind}_{B}^{G}(\tau(\Lambda) \otimes(-\lambda) \otimes 1)_{K \text {-finite. So }}$

$$
B_{\lambda} f=\beta_{\lambda} P_{\lambda}(T \phi)=C(\lambda) T \pi \neq 0 .
$$

This contradicts the assumption $f \in \mathfrak{W} \subset \operatorname{ker}\left(\beta_{\lambda}\right)$.

\section{VeCTOR-VALUED Distributions}

Suppose $K$ is a Lie group and $V$ a finite dimensional space over $\mathbb{C}$. Let $C^{-\infty}(K, V)$ denote all continuous $\mathbb{C}$-linear maps from $C_{c}^{\infty}(K, \mathbb{C})$ to $V$. Let $M$ be a compact subgroup of $K$, and $(\pi, V)$ a finite dimensional representation of $M$. Let $C^{-\infty} \operatorname{Ind}_{M}^{K}(\pi)$ be the space defined by

$$
\left\{f \in C^{-\infty}(K, V) \mid R_{m} f(\phi)=\pi\left(m^{-1}\right) f(\phi), \forall \phi \in C_{c}^{\infty}(K, \mathbb{C}), \forall m \in M\right\} .
$$

Here $R_{m} f(\phi)=f\left(R_{m^{-1}} \phi\right)$, with $R_{m^{-1}} \phi(k)=\phi\left(k m^{-1}\right)$.

Let $\left(\check{\pi}, V^{*}\right)$ be the dual representation of $(\pi, V)$, and $\langle$,$\rangle the nondegenerate$ bilinear form on $V \times V^{*}$. Let $\left(C_{c}^{\infty} \operatorname{Ind}_{M}^{K}(\pi)\right)^{\prime}$ be the strong dual of $C_{c}^{\infty} \operatorname{Ind}_{M}^{K}(\pi)$. For each $T \in\left(C_{c}^{\infty} \operatorname{Ind}_{M}^{K}(\pi)\right)^{\prime}, \phi \in C_{c}^{\infty}(K, \mathbb{C})$, and $v \in V$, we define $\xi_{1}(T)(\phi) \in V^{*}$ by

$$
\left\langle v, \xi_{1}(T)(\phi)\right\rangle=T\left(\xi_{1}(\phi, v)\right)
$$

where $\xi_{1}(\phi, v)(k)=\int_{M} \phi(k m) \pi(m) v d m$. It is easy to show that

$$
\xi_{1}(T) \in C^{-\infty} \operatorname{Ind}_{M}^{K}(\check{\pi}) .
$$

Proposition 9.1. The map $\xi_{1}:\left(C_{c}^{\infty} \operatorname{Ind}_{M}^{K}(\pi)\right)^{\prime} \rightarrow C^{-\infty} \operatorname{Ind}_{M}^{K}(\check{\pi})$ is bijective.

Proof. Define

$$
\eta_{1}: C^{-\infty} \operatorname{Ind}_{M}^{K}(\check{\pi}) \rightarrow\left(C_{c}^{\infty} \operatorname{Ind}_{M}^{K}(\pi)\right)^{\prime}
$$

as follows: for each $f \in C^{-\infty} \operatorname{Ind}_{M}^{K}(\check{\pi})$, and $\phi \in C_{c}^{\infty} \operatorname{Ind}_{M}^{K}(\pi)$, the map

$$
f_{\phi}: u \rightarrow f(\langle\phi, u\rangle)
$$

is a linear map from $V^{*}$ to $V^{*}$. Then we define

$$
\eta_{1}(f)=\operatorname{tr}\left(f_{\phi}\right) \text {. }
$$

It is a long but rather straightforward calculation to show $\xi_{1}$ and $\eta_{1}$ are inverses to each other.

Now let $G=K A N$, and $\left(\delta, V_{\delta}\right)$ be a finite dimensional representation of $B=$ $M A N$. Let

$$
\begin{gathered}
C^{\infty} \operatorname{Ind}_{B}^{G}(\delta)=\left\{f \in C^{\infty}\left(G, V_{\delta}\right) \mid R_{\text {man }} f=a^{-\rho} \delta^{-1}(\text { man }) f, \forall \text { man } \in B\right\}, \\
C^{-\infty} \operatorname{Ind}_{B}^{G}(\delta)=\left\{f \in C^{-\infty}\left(G, V_{\delta}\right) \mid R_{\text {man }} f=a^{-\rho} \delta^{-1}(\text { man }) f, \forall \text { man } \in B\right\} .
\end{gathered}
$$

For $T \in\left(C^{\infty} \operatorname{Ind}_{B}^{G}(\delta)\right)^{\prime}, \xi(T)$ is defined by

$$
\langle v, \xi(T)(\phi)\rangle=T(\xi(\phi, v)),
$$


for each $v \in V_{\delta}$, and $\phi \in C_{c}^{\infty}(G, \mathbb{C})$. Here $\xi(\phi, v)$ is defined as follows: for each $g \in G$,

$$
\xi(\phi, v)(g)=\int_{M A N} \phi(\operatorname{gman}) a^{\rho} \delta(\operatorname{man}) v d m d a d n .
$$

Now we show $\xi(T) \in C^{-\infty} \operatorname{Ind}_{B}^{G}(\check{\delta})$. By definition,

$$
\left\langle v, \xi(T)\left(R_{(\operatorname{man})^{-1}} \phi\right)\right\rangle=T\left(\xi\left(R_{(\operatorname{man})^{-1}} \phi, v\right)\right) .
$$

However, it is a simple calculation to see

$$
\xi\left(R_{(\operatorname{man})^{-1}} \phi, v\right)=\xi\left(\phi, a^{-\rho} \delta(\operatorname{man}) v\right) .
$$

Hence

$$
\begin{aligned}
\left\langle v, R_{\operatorname{man}} \xi(T)(\phi)\right\rangle & =\left\langle v, \xi(T)\left(R_{\left.(\operatorname{man})^{-1} \phi\right)}\right\rangle\right. \\
& =T\left(\xi\left(\phi, a^{-\rho} \delta(\operatorname{man}) v\right)\right) \\
& =\left\langle a^{-\rho} \delta(\operatorname{man}) v, \xi(T)(\phi)\right\rangle \\
& =\left\langle v, a^{-\rho} \check{\delta}\left((\operatorname{man})^{-1}\right) T(\phi)\right\rangle .
\end{aligned}
$$

This proves $\xi(T) \in C^{-\infty} \operatorname{Ind}_{B}^{G}(\check{\delta})$.

Theorem 9.2. Let $\xi$ be defined as above. Then $\xi$ is a $G$-equivariant bijection from $\left(C^{\infty} \operatorname{Ind}_{B}^{G}(\delta)\right)^{\prime}$ to $C^{-\infty} \operatorname{Ind}_{B}^{G}(\check{\delta})$.

Lemma 9.3. Let $L$ be a Lie group and $(\pi, V)$ a finite dimensional representation of $L$ on $V$. Suppose $f \in C^{-\infty}(L, V)$, satisfying

$$
R_{l} f=\pi\left(l^{-1}\right) f,
$$

for each $l \in L$. Let $d l$ be the right invariant Haar measure on $L$. Then there exists a unique vector $v \in V$, such that

$$
f(\phi)=\int_{L} \phi(l) \pi\left(l^{-1}\right) v d l,
$$

for each $\phi \in C_{c}^{\infty}(L, \mathbb{C})$.

Proof. We use an argument due to Helgason. For $\phi$ and $\psi$ in $C_{c}^{\infty}(L, \mathbb{C})$, we define $\phi * \psi$ in $C_{c}^{\infty}(L, \mathbb{C})$ by

$$
\phi * \psi(x)=\int_{L} \phi(l) \psi\left(x l^{-1}\right) d l .
$$

Then

$$
f(\phi * \psi)=\int_{L} \phi(l) f\left(R_{l^{-1}} \psi\right) d l=\int_{L} \phi(l) \pi\left(l^{-1}\right) f(\psi) d l .
$$

Choose a sequence $\psi_{n}$ such that $\check{\psi}_{n} \rightarrow \delta$, the delta function, as $n \rightarrow+\infty$. Here $\check{\psi}_{n}(l)=\psi_{n}\left(l^{-1}\right)$. Let $v_{n}=f\left(\psi_{n}\right)$. Then

$$
f\left(\phi * \psi_{n}\right)=\int_{L} \phi(l) \pi\left(l^{-1}\right) v_{n} d l .
$$

We can choose an appropriate $\phi$ (e.g. close to $\delta$ ), such that $\int_{L} \phi(l) \pi\left(l^{-1}\right) d l$ is invertible. Since $\phi * \psi_{n} \rightarrow \phi$, by letting $n \rightarrow+\infty$ in (*), we conclude there exists $v \in V$, such that $v_{n} \rightarrow v$, and

$$
f(\phi)=\int_{L} \phi(l) \pi\left(l^{-1}\right) v d l
$$


The uniqueness follows from the fact that there is $\phi$ such that $\int_{L} \phi(l) \pi\left(l^{-1}\right) d l$ is invertible.

Proof of Theorem 9.2. First we construct the inverse $\eta$ of $\xi$ as follows: Take $f \in$ $C^{-\infty} \operatorname{Ind}_{B}^{G}(\check{\delta})$, and $\psi \in C^{\infty}(K, \mathbb{C})$. Then $\phi \rightarrow f(\psi \otimes \phi)$ defines a continuous linear map from $C_{c}^{\infty}(A \times N, \mathbb{C})$ to $V_{\delta}^{*}$, where

$$
(\psi \otimes \phi)(k a n)=\psi(k) \phi(a n) .
$$

It is easy to check this map satisfies all the conditions as in Lemma 9.3 if we take $L=A N, \pi(a n)=a^{\rho} \check{\delta}($ an $)$. So there exists a unique element in $V_{\delta}^{*}$, which we denote by $f^{-}(\psi)$, such that

$$
f(\psi \otimes \phi)=\int_{A \times N} \phi(a n) a^{\rho} \check{\delta}^{-1}(a n) f^{-}(\psi) d a d n .
$$

Notice $a^{2 \rho} d a d n$ gives a right invariant Haar measure on $A N$. It is fairly easy to see $f^{-} \in C^{-\infty} \operatorname{Ind}_{M}^{K}(\check{\delta} \mid M)$. Then by Proposition $9.1 \eta_{1}\left(f^{-}\right)$gives an element in $\left(C^{\infty} \operatorname{Ind}_{M}^{K}(\delta \mid M)\right)^{\prime}$. Since $C^{\infty} \operatorname{Ind}_{M}^{K}(\delta \mid M) \cong C^{\infty} \operatorname{Ind}_{B}^{G}(\delta)$, one can view $\eta_{1}\left(f^{-}\right)$as an element in $\left(C^{\infty} \operatorname{Ind}_{B}^{G}(\delta)\right)^{\prime}$. Finally we define $\eta(f)$ by

$$
\eta(f)=\eta_{1}\left(f^{-}\right) .
$$

The final step of the proof is to show $\eta \circ \xi=\mathrm{id}$, and $\eta \circ \xi=$ id. For each $T \in\left(C^{\infty} \operatorname{Ind}_{B}^{G}(\delta)\right)^{\prime}, \psi \in C^{\infty}(K, \mathbb{C})$, and $\phi \in C_{c}^{\infty}(A \times N, \mathbb{C})$,

$$
\xi(T)(\psi \otimes \phi)=\int_{A \times N} \phi(a n) a^{\rho} \check{\delta}^{-1}(a n)(\xi(T))^{-} d a d n .
$$

So for each $v \in V$,

$$
(* *) \quad\langle v, \xi(T)(\psi \otimes \phi)\rangle=\left\langle v, \int_{A \times N} \phi(a n) a^{\rho} \check{\delta}^{-1}(a n)(\xi(T))^{-}(\psi) d a d n\right\rangle .
$$

By definition

$$
\begin{aligned}
\xi(\psi \otimes \phi, v)(k) & =\int_{M A N}(\psi \otimes \phi)(k m a n) a^{\rho} \delta(\operatorname{man}) v d m d a d n \\
& =\int_{M A N} \psi(k m) \delta(m) \phi(a n) a^{\rho} \delta(a n) v d m d a d n \\
& =\xi_{1}\left(\psi, v_{1}\right),
\end{aligned}
$$

where $v_{1}=\int_{A \times N} a^{\rho} \phi(a n) \delta(a n) v d a d n$. So by $(* *)$

$$
\begin{aligned}
\langle v, \xi(T)(\psi \otimes \phi)\rangle & =T\left(\xi_{1}\left(\psi, v_{1}\right)\right) \\
& =\left\langle v_{1}, \xi_{1}(T)(\psi)\right\rangle \\
& =\left\langle v, \int_{A \times N} \phi(a n) a^{\rho} \check{\delta}^{-1}(a n) \xi_{1}(T)(\psi) d a d n\right\rangle .
\end{aligned}
$$

Hence

$$
\begin{aligned}
\int_{A \times N} \phi(a n) a^{\rho} \check{\delta}^{-1}(a n)(\xi(T))^{-}(\psi) d a d n \\
\left.=\int_{A \times N} \phi(a n) a^{\rho} \check{\delta}^{-1}(a n) \xi_{1}(T)(\psi) d a d n\right\rangle .
\end{aligned}
$$

By comparing both sides we have $\xi_{1}(T)=(\xi(T))^{-}$. Hence

$$
T=\xi_{1}^{-1}\left((\xi(T))^{-}\right)=\eta_{1}\left((\xi(T))^{-}\right)=\eta(\xi(T)) .
$$


Similarly we can verify $\xi \circ \eta=$ id. Note it is enough to check on functions of the form $\psi \otimes \phi$.

Now suppose $V_{\delta}$ is a Hilbert space. Let $\delta^{*}$ be the representation defined as follows: for each $g \in G, w, v \in V_{\delta}$, we have $\langle\delta(g) v, w\rangle=\left\langle v, \delta(g)^{t} w\right\rangle$; then $\delta^{*}(g)=$ $\delta\left(g^{-1}\right)^{t}$. Let $C^{-\infty} \operatorname{Ind}_{B}^{G}\left(\delta^{*}\right)$ be the space of conjugate linear maps $f$ from $C_{c}^{\infty}(G, \mathbb{C})$ to $V_{\delta}$, such that

$$
R_{\text {man }} f=a^{-\rho} \delta^{*}\left((\operatorname{man})^{-1}\right) f .
$$

For each $T \in\left(C^{\infty} \operatorname{Ind}_{B}^{G}(\delta)\right)^{\prime}$, and $\phi \in C_{c}^{\infty}(G, \mathbb{R}), \xi(T)(\phi)$ is defined by

$$
\langle v, \xi(T)(\phi)\rangle=T(\xi(\phi, v)),
$$

for each $v \in V_{\delta}$. Here

$$
\xi(\phi, v)(g)=\int_{M A N} \phi(\operatorname{gman}) a^{\rho} \delta(\operatorname{man}) v d m d a d n .
$$

Corollary 9.4. $\xi$ is a bijection from $\left(C^{\infty} \operatorname{Ind}_{B}^{G}(\delta)\right)^{\prime}$ onto $C^{-\infty} \operatorname{Ind}_{B}^{G}\left(\delta^{*}\right)$.

Let $\sigma$ be a unitary representation of $M$ and $\lambda \in \mathfrak{a}_{\mathbb{C}}^{*} \cdot \sigma \otimes \bar{\lambda} \otimes 1$ is the representation of $B$ defined by $\operatorname{man} \rightarrow a^{\bar{\lambda}} \sigma(m)$. Then

$$
(\sigma \otimes \bar{\lambda} \otimes 1)^{*}=\sigma \otimes(-\lambda) \otimes 1 .
$$

Corollary 9.5. The map

$$
\xi:\left(C^{\infty} \operatorname{Ind}_{B}^{G}(\sigma \otimes \bar{\lambda} \otimes 1)\right)^{\prime} \rightarrow C^{-\infty} \operatorname{Ind}_{B}^{G}(\sigma \otimes(-\lambda) \otimes 1)
$$

is a bijection.

\section{Distribution Boundary VAlues}

We now introduce a weak growth condition in the eigenspace $\mathcal{E}_{\lambda-\Lambda} \operatorname{Ind}_{K}^{G}(\tau)$. Recall in Section 2 we have

$$
C_{r}^{q}(G, V)=\left\{f \in C^{q}(G, V)\|\| f \|_{q, r}<\infty\right\},
$$

$q \in \mathbb{N}$ and $r \in \mathbb{R}$. $C_{r}^{\infty}(G, V)=\bigcap_{q} C_{r}^{q}(G, V)$. We define $\mathfrak{F}$ to be the space

$$
\mathfrak{F}=\bigcap_{r} C_{r}^{\infty}(G, V)=\bigcap_{q, r} C_{r}^{q}(G, V),
$$

endowed with the projective limit topology for the intersection over $q$ and $r$ (i.e., the topology given by the family of forms $\|\cdot\|_{q, r}$ ). Using the same argument as on p. 142 in [BS] we conclude $\mathfrak{F}$ is a Fréchet space. It follows from Section 2 that $L$ and $R$ act smoothly on $\mathfrak{F}$.

Let $\mathfrak{F}^{\prime}$ be the space dual to $\mathfrak{F}$, equipped with the strong dual topology. For each $T \in \mathfrak{F}^{\prime}, q \in \mathbb{N}$, and $r \in \mathbb{R}$, we define

$$
\|T\|_{q, r}^{\prime}=\sup \left\{T(\varphi) \mid \varphi \in \mathfrak{F},\|\varphi\|_{q, r} \leq 1\right\} .
$$

The space $C_{r}^{q}(G, V)^{\prime}=\left\{T \in \mathfrak{F}^{\prime} \mid\|T\|_{q, r}^{\prime}<\infty\right\}$ with this norm is the dual space of $C_{r}^{q}(G, V)$. Moreover, we have $\mathfrak{F}^{\prime}=\bigcup_{q, r} C_{r}^{q}(G, V)^{\prime}$. By duality $\mathfrak{F}^{\prime}$ is the inductive limit of these spaces. Using Lemma 2.1 we can prove that for some $b \in \mathbb{R}$, $\int_{G}\|g\|^{b} d g<\infty$. It follows that there is a continuous injection of $C_{r}^{0}(G, V)$ into $C_{b-r}^{0}(G, V)^{\prime}$ defined by integration over $G$. Hence there is a continuous injection of $C_{r}^{0}(G, V)$ into $\mathfrak{F}^{\prime}$. 
Let $q^{\prime} \geq q$, and $r \in \mathbb{R}$. For each $T \in C_{r}^{q}(G, V)^{\prime}$, and $\varphi \in C_{r}^{q^{\prime}}(G, \mathbb{R})$, we define an element $L^{\vee}(\varphi) T$ in $C_{r}^{q^{\prime}-q}(G, V)$ by

$$
\left\langle v, L^{\vee}(\varphi) T(x)\right\rangle=T\left(R_{x^{-1}} \varphi \cdot v\right) .
$$

Note if $f \in C_{r}^{0}(G, V)$, and $\varphi \in C_{b-r}^{0}(G, \mathbb{C})$, then

$$
L^{\vee}(\varphi) f(x)=\int_{G} \varphi(g) f(g x) d g .
$$

Lemma 10.1. Let $q, q^{\prime} \in \mathbb{N}$ with $q \leq q^{\prime}$. There exist $s \geq 0$ and $C \geq 0$ such that

$$
\left\|L^{\vee}(\varphi) T\right\|_{q^{\prime}-q, r} \leq C\|T\|_{q^{\prime}, r}^{\prime}\|\varphi\|_{q^{\prime}, r-s},
$$

for all $r \in \mathbb{R}, T \in C_{r}^{q}(G, V)^{\prime}$, and $\varphi \in C_{r-s}^{q^{\prime}}(G, \mathbb{R})$.

Proof. See Lemma 11.1 in [BS].

Let $\mathcal{E}_{\lambda-\Lambda}^{*} \operatorname{Ind}_{K}^{G}(\tau)$ denote the closed subspace $\mathcal{E}_{\lambda-\Lambda} \operatorname{Ind}_{K}^{G}(\tau) \cap \mathfrak{F}^{\prime}$. We call the elements of $\mathcal{E}_{\lambda-\Lambda}^{*} \operatorname{Ind}_{K}^{G}(\tau)$ eigensections of weak moderate growth. Notice if $f \in$ $\mathcal{E}_{\lambda-\Lambda}^{*} \operatorname{Ind}_{K}^{G}(\tau)$, and $\varphi \in C_{c}^{\infty}(C, \mathbb{R})$, then $L^{\vee}(\varphi) f \in \mathcal{E}_{\lambda-\Lambda}^{\infty} \operatorname{Ind}_{K}^{G}(\tau)$ by Lemma 10.1. For $\lambda-\mathrm{E} \in \mathfrak{A}_{1}, \lambda \in \mathfrak{A}_{2}$, and $f \in \mathcal{E}_{\lambda-\Lambda}^{*} \operatorname{Ind}_{K}^{G}(\tau)$, we define a vector-valued distribution $\bar{\beta}_{\lambda} f$ on $G$ by

$$
\bar{\beta}_{\lambda} f(\varphi)=\beta_{\lambda}\left(L^{\vee}(\varphi) f\right)(e),
$$

for each $\varphi \in C_{c}^{\infty}(G, \mathbb{R})$.

Proposition 10.2. $\bar{\beta}_{\lambda}$ is a linear, continuous, and G-equivariant map from $\mathcal{E}_{\lambda-\Lambda}^{*} \operatorname{Ind}_{K}^{G}(\tau)$ to $C^{-\infty} \operatorname{Ind}_{B}^{G}(\tau(\Lambda) \otimes(-\lambda) \otimes 1)$.

Proof. It suffices to show $\bar{\beta}_{\lambda} f \in C^{-\infty} \operatorname{Ind}_{B}^{G}(\tau(\Lambda) \otimes(-\lambda) \otimes 1)$. By definition,

$$
\begin{aligned}
L^{\vee}\left(R_{(\operatorname{man})^{-1}} \varphi\right) f(x) & =f\left(R_{x^{-1}} R_{(\operatorname{man})^{-1}} \varphi\right) \\
& =f\left(R_{(\operatorname{man} x)^{-1}} \varphi\right) \\
& =L^{\vee}(\varphi) f(\operatorname{man} x) .
\end{aligned}
$$

However, $\beta_{\lambda}$ is $G$-equivariant. Hence

$$
\begin{aligned}
B_{\lambda}\left(L^{\vee}\left(R_{(\operatorname{man})^{-1}} \varphi\right) f\right)(e) & =\beta_{\lambda}\left(L^{\vee}(\varphi) f\right)(\operatorname{man}) \\
& =\tau(\Lambda)\left(m^{-1}\right) a^{\lambda-\rho} \beta_{\lambda}\left(L^{\vee}(\varphi) f\right)(e) .
\end{aligned}
$$

This proves $\bar{\beta}_{\lambda} f \in C^{-\infty} \operatorname{Ind}_{B}^{G}(\tau(\Lambda) \otimes(-\lambda) \otimes 1)$.

For each $T \in\left(C^{\infty} \operatorname{Ind}_{B}^{G}(\tau(\Lambda) \otimes \bar{\lambda} \otimes 1)\right)^{\prime}$, we define $\bar{P}_{\lambda} T$ as follows:

$$
\left.\left\langle v, \bar{P}_{\lambda} T(g)\right\rangle=T\left(P(\Lambda) L_{g} \Phi_{\lambda} \cdot v\right)\right\rangle
$$

for each $v \in V$. Here $\Phi_{\lambda}(x)$ is the transpose of $\Psi_{\lambda}\left(x^{-1}\right)$, and $P(\Lambda)$ the projection from $V$ to $V(\Lambda)$. The motivation of this definition is from Corollary 1.10.

Proposition 10.3. $\bar{P}_{\lambda} T \in \mathcal{E}_{\lambda-\Lambda}^{*} \operatorname{Ind}_{K}^{G}(\tau)$, for $T \in\left(C^{\infty} \operatorname{Ind}_{B}^{G}(\tau(\Lambda) \otimes \bar{\lambda} \otimes 1)\right)^{\prime}$. And $\bar{P}_{\lambda}$ is linear, continuous, and $G$-equivariant.

Proof. Similar to the proof for Corollary 11.3 in [BS].

Lemma 10.4. Let $T \in\left(C^{\infty} \operatorname{Ind}_{B}^{G}(\tau(\Lambda) \otimes \bar{\lambda} \otimes 1)\right)^{\prime}$, and $\varphi \in C_{c}^{\infty}(G, \mathbb{R})$. Then $L^{\vee}(\varphi) \bar{P}_{\lambda} T=P_{\lambda}\left(L^{\vee}(\varphi) \xi(T)\right)$. Here $\xi$ is the isomorphism in Corollary 9.5, and $L^{\vee}(\varphi) \xi(T)(x)=\xi(T)\left(R_{x^{-1}} \varphi\right)$. 
Proof. $L^{\vee}(\varphi), \bar{P}_{\lambda}$, and $P_{\lambda}$ are continuous. So it is enough to check for $T \in$ $C^{\infty} \operatorname{Ind}_{B}^{G}(\tau(\Lambda) \otimes(-\lambda) \otimes 1)$. The proof follows from the $G$-equivariance of $P_{\lambda}$.

By a similar argument we get

Lemma 10.5. Let $f \in \mathcal{E}_{\lambda-\Lambda}^{*} \operatorname{Ind}_{K}^{G}(\tau)$, and $\varphi \in C_{c}^{\infty}(G, \mathbb{R})$. Then

$$
L^{\vee}(\varphi) \bar{\beta}_{\lambda} f=\beta_{\lambda}\left(L^{\vee}(\varphi) f\right) .
$$

Theorem 10.6. Under the same conditions as in Theorem 8.3, $\bar{P}_{\lambda}$ defines a G-equivariant topological isomorphism from $\left(C^{\infty} \operatorname{Ind}_{B}^{G}(\tau(\Lambda) \otimes \bar{\lambda} \otimes 1)\right)^{\prime}$ onto $\mathcal{E}_{\lambda-\Lambda}^{*} \operatorname{Ind}_{K}^{G}(\tau)$. And $\eta \circ C_{0}(\lambda)^{-1} \circ \bar{\beta}_{\lambda}$ gives the inverse of $\bar{P}_{\lambda}$.

Proof. By Theorem 8.1 and Lemma 10.4, 10.5,

$$
L^{\vee}(\varphi) \bar{\beta}_{\lambda} \bar{P}_{\lambda} T=\beta_{\lambda} P_{\lambda} L^{\vee}(\varphi) \xi(T)=C_{0}(\lambda) L^{\vee}(\varphi) \xi(T),
$$

for $T \in\left(C^{\infty} \operatorname{Ind}_{B}^{G}(\tau(\Lambda) \otimes \bar{\lambda} \otimes 1)\right)^{\prime}$. Similarly, for each $f \in \mathcal{E}_{\lambda-\Lambda}^{*} \operatorname{Ind}_{K}^{G}(\tau)$,

$$
L^{\vee}(\varphi) \bar{P}_{\lambda} \eta\left(C_{0}(\lambda)^{-1} \bar{\beta}_{\lambda} f\right)=P_{\lambda} C_{0}(\lambda)^{-1} \beta_{\lambda} L^{\vee}(\varphi) f=L^{\vee}(\varphi) f .
$$

So we have

$$
\bar{\beta}_{\lambda} \circ \bar{P}_{\lambda}=C_{0}(\lambda) \circ \xi, \quad \bar{P}_{\lambda} \circ \eta \circ C_{0}(\lambda)^{-1} \bar{\beta}_{\lambda}=\mathrm{id} .
$$

Remark 10.7. Let $\mathcal{E}_{\lambda-\Lambda, r} \operatorname{Ind}_{K}^{G}(\tau)=\mathcal{E}_{\lambda-\Lambda} \operatorname{Ind}_{K}^{G}(\tau) \cap C_{r}(G, V)$ be equipped with the Banach space topology inherited from $C_{r}(G, V)$. Then $\mathcal{E}_{\lambda-\Lambda}^{*} \operatorname{Ind}_{K}^{G}(\tau)$ is identical with the inductive limit topology for the union $\mathcal{E}_{\lambda-\Lambda}^{*} \operatorname{Ind}_{K}^{G}(\tau)=\bigcup_{r} \mathcal{E}_{\lambda-\Lambda, r} \operatorname{Ind}_{K}^{G}(\tau)$. See p. 146 in [BS].

A classical result asserts that the left $K$-finite elements in $\mathcal{E}_{\lambda-\Lambda} \operatorname{Ind}_{K}^{G}(\tau)$ increase at most exponentially. So by the remark above we easily get

Corollary 10.8. Under the same conditions as in Theorem 8.3, $P_{\lambda}$ is a bijection from $C^{\infty} \operatorname{Ind}_{B}^{G}\left(\tau(\Lambda) \otimes\left({ }_{\lambda}\right) \otimes 1\right)_{K \text {-finite }}$ to $\mathcal{E}_{\lambda-\Lambda} \operatorname{Ind}_{K}^{G}(\tau)_{K \text {-finite }}$.

Remark 10.9. I think by Schmid's method indicated in [Sch] one should be able to get a bijection on the level of hyperfunctions from Corollary 10.8.

\section{ACKNOWLEDGEMENT}

The paper is part of the author's thesis. He is very grateful to Professor Sigurdur Helgason for his guidance and encouragement. He also would like to thank Professor David Vogan for his generous help.

\section{REFERENCES}

[Ban] E. P. van den Ban, The principal series for a reductive symmetric space II, Eisenstein integrals, J. Funct. Anal. 109 (1992), 331-441. MR 93j:22025

[BS] E. P. van den Ban, H. Schlichtkrull, Asymptotic expansions and boundary values of eigenfunctions on Riemannian symmetric spaces, J. Reine Angew. Math. 380 (1987), 108-165. MR 89g:43010

[Ca] W. Casselman, Canonical extensions of Harish-Chandra modules to representations of G, Canad. J. Math. (1989), 385-438. MR 90j:22013

[Deit] A. Deitmar, Invariant operators on higher $K$-types, J. Reine Angew. Math. 412 (1990), 97-107. MR 92b:22013

[Ga] P. Gaillard, Eigenforms of the Laplacian on real and complex hyperbolic spaces, J. Funct. Anal. 78 (1988), 99-115. MR 89h:58198

[Helg1] S. Helgason, Group and Geometric Analysis, Academic Press, 1984. MR 86c:22017 
[Helg2] _ A duality for symmetric spaces with applications to group representations I, II, Adv. in Math. 5 (1970), 1-154; 22 (1976), 1187-219. MR 55:3169

[Helg3] Some results on invariant differential operators on symmetric spaces, Amer. J. Math. 114 (1992), 789-811. MR 94a:22020

[Kato] T. Kato, Perturbation theory for linear operators, 1980.

[KKMOOT] M. Kashiwara, A. Kowata, K. Minemura, K. Okamoto, T. Oshima, M. Tanaka, Eigenfunctions of invariant differential operators on a symmetric space, Ann. of Math. (2) 107 (1978), 1-39. MR 81f:43013

[Lep] J. Lepowsky, Algebraic results on representations of semisimple Lie groups, Trans. Amer. Math. Soc. 176 (1973), 1-44. MR 49:10819

[Lew] J. Lewis, Eigenfunctions on symmetric spaces with distribution valued boundary forms, J. Funct. Anal. 29 (1978), 331-357. MR 80f:43020

[Min] K. Minemura, Invariant differential operators and spherical sections of a homogeneous vector bundle, Tokyo J. Math. 15 (1992), 231-245. MR 93b:22014

[OS] T. Oshima, J. Sekiguchi, Eigenspaces of invariant differential operators on an affine symmetric space, Invent. Math. 57 (1980), 1-81. MR 81k:43014

[Shim] N. Shimeno, Eigenspaces of invariant differential operators on a homogeneous line bundle on a Riemannian symmetric space, J. Fac. Sci. Univ. Tokyo Sect. IA Math. 37 (1990), 201-234. MR 91j:22008

[Sch] W. Schmid, Boundary value problems for group invariant differential equations, Astérique, hors série, 1985, 311-321. MR 87h:22018

[SV] B. Speh, D. Vogan, Reducibility of generalized principal series representations, Acta Math. 145 (1980), 227-299. MR 82c:22018

[Ven] H. van der Ven, Vector valued Poisson transforms on Riemannian symmetric spaces of rank one, J. Funct. Anal. 119 (1994), 358-400. MR 96a:22016

[Vogan] D. Vogan, Representations of Real Reductive Lie Groups, Birkhäuser, 1981. MR 83c:22022

[Wall] N. Wallach, Real Reductive Groups. I, II, Academic Press, 1988 \& $1992 . \quad$ MR 89i:22029; MR 93m:22018

[Wall1] N. Wallach, Asymptotic expansions of generalized matrix entries of representations of real reductive groups, Lie Group Representations, I. Lecture Notes in Mathematics, 1024, Springer-Verlag, 1983. MR 85g:22029

[Wall2] N. Wallach, On Harish-Chandra's generalized $C$-functions, Amer. J. Math. 97 (1975), 386-403. MR 53:3202

[War] G. Warner, Harmonic Analysis on Semisimple Lie Groups. I, II, 1972. MR 58:16979; MR 58:16980

Department of Mathematics, Massachusetts Institute of Technology, 2-251, CamBRIDGe, Massachusetts 02139

Current address: Micro Strategy, 5th Floor, 2650 Park Tower Dr., Metro Place 1, Vienna, Virginia 22180

E-mail address: yang@strategy.com 